\title{
QUANTUM NONLINEAR OPTICS
}

\author{
Thesis \\ Submitted to \\ The School of Engineering of the \\ UNIVERSITY OF DAYTON
}

In Partial Fulfillment of the Requirements for

The Degree of

Master of Science in Electro-Optics

\author{
By \\ Xuesong Gao \\ UNIVERSITY OF DAYTON \\ Dayton, Ohio
}

August 2019 


\section{QUANTUM NONLINEAR OPTICS}

Name: Gao, Xuesong

APPROVED BY:

Imad Agaha, Ph.D.

Advisory Committee Chairman

Assistant Professor

Electro-Optics and Photonics
Chenglong Zhao, Ph.D.

Committee Member

Assistant Professor

Electro-Optics and Photonics and Department of Physics

Bill Plick, Ph.D.

Committee Member

Assistant Professor

Electro-Optics and Photonics and

Department of Physics

Robert J. Wilkens, Ph.D., P.E

Associate Dean, Research and Innovation

Professor

School of Engineering
Eddy M. Rojas, Ph.D., M.A., P.E.

Dean, School of Engineering 
(C) Copyright by

Xuesong Gao

All rights reserved

2019 


\section{ABSTRACT \\ QUANTUM NONLINEAR OPTICS}

Name: Gao, Xuesong

University of Dayton

Advisor: Imad Agha, Ph.D.

Quantum computing research has been around for several decades, since it was first proposed by Richard Feynman. Many efforts have been made to utilize quantum systems in creation of new computing devices in the recent years. One of the essential tools used for characterization and manipulation of optical quantum systems is a Hong-Ou-Mandel (HOM) Interferometer. It is used in this work to provide information about propagation, distortion and degree of indistinguishability of single photons.

This work can be also seen as the transition from the classic nonlinear optics to the quantum nonlinear optics. We provide a necessary basic nonlinear background, such as phase matching and parametric down conversion process. Then we introduce the phenomenon of the HOM effect and HOM dip on the base of the photon entanglement and photon antibunching process.

The HOM interferometer is also used to measure the correlations between two coherent photon sources. Single photons generated by parametric down conversion are tailored through aggressive filtering techniques to be identical in their spectral, temporal and polarization profiles. The visibility of the correlation photons is a judgement to measure whether the photons can be distinguished or not. 
This work attempts to provide better understanding of single photon interactions by SPDC process through use of an HOM interferometer.

Key words: Parametric down conversion, HOM interferometer, HOM dip and visibility. 
Dedicated To My Parents,

For all the support that they gave me to reach this level 


\section{ACKNOWLEDGEMENTS}

I would like to thank my advisor Dr Imad Agha and Dr. Joseph W. Haus, for their patience, advice, guidance and support. I would also like to thank my committee members Dr. Chenglong Zhao and Dr. Bill Plick for their support and helpful suggestions.

I would like to specially thank Dr. Imad Agha for the support throughout my thesis work and give me the right suggestions on my studies. I also would like to specially thank Mallik Mohd Raihan Hussain for spending his valuable time in the lab with me helping on my simulation and helping in editing my thesis. I would like to thank my friends and fellow students for their support in this journey.

I would like to thank Dr. Partha Banerjee and Dr. Rita Peterson for their classes and advice. Also, I need give a special thanks to Dr. Chenglong Zhao for his advice on studies and on extracurricular activities.

Finally, I would like to specially thank my parents for supporting me all the time. 


\section{TABLE OF CONTENTS}

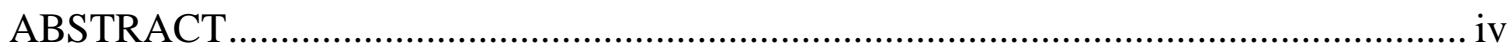

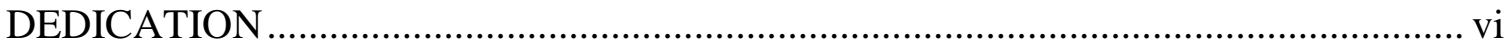

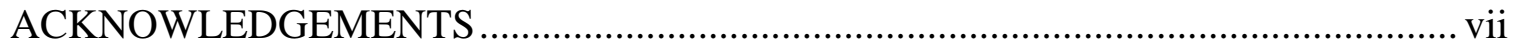

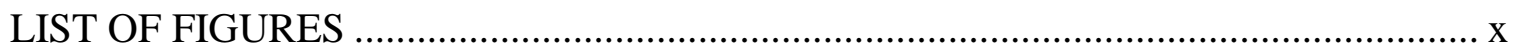

LIST OF ABBREVIATIONS .............................................................................. xii

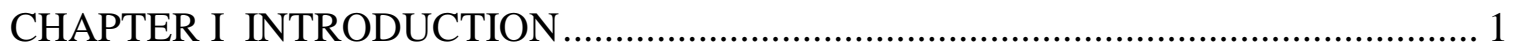

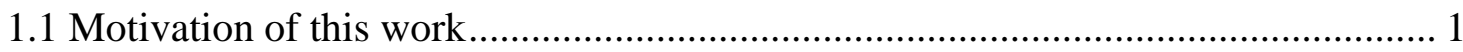

1.2 Structure of this work .................................................................................. 2

CHAPTER II NONLINEAR PARAMETRIC PROCESS AND PHASE MATCHING . 5

2.1 Nonlinear parametric processes .............................................................. 5

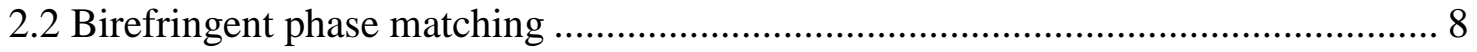

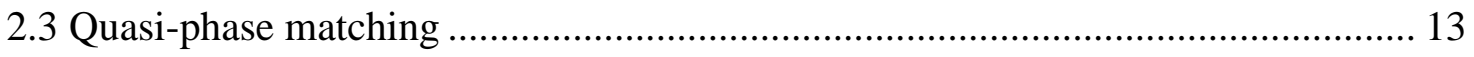

2.4 Spontaneous parametric down conversion (SPDC) ..................................... 16

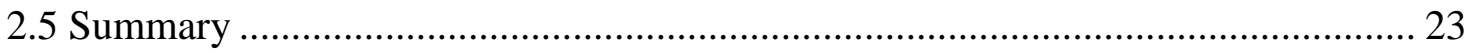

CHAPTER III HOM INTERFEROMETER AND SINGLE PHOTON SOURCES ...... 24

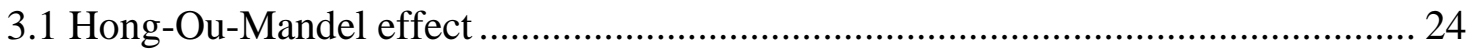

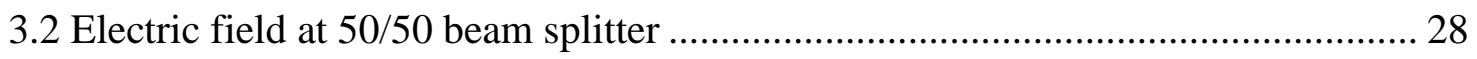

3.3 HOM interferometer.................................................................................. 32 
3.4 Degree of coherence and photon antibunching 34

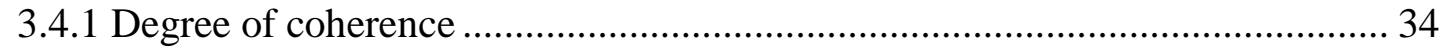

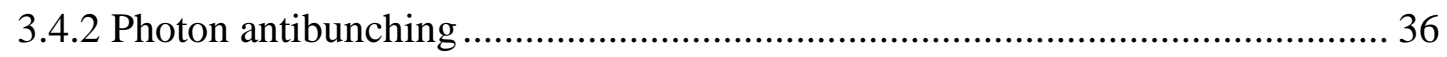

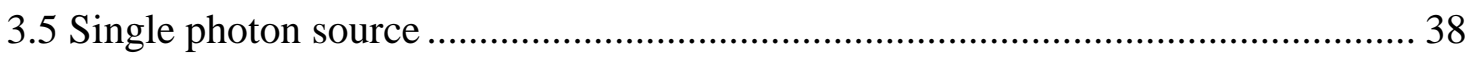

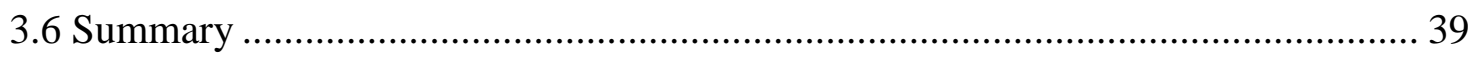

CHAPTER IV MANDEL DIP EXPERIMENT AND HOM VISIBILITY ................... 40

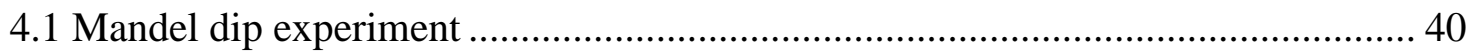

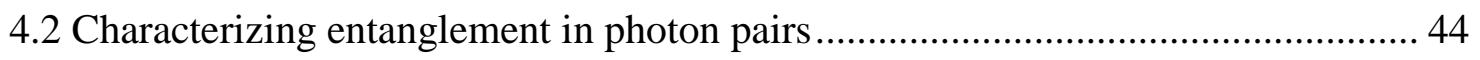

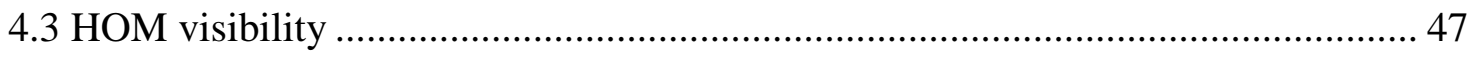

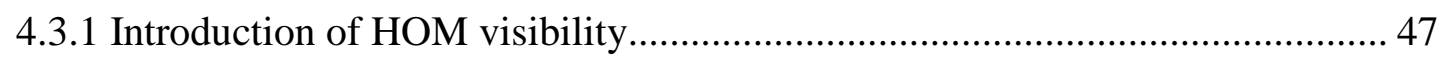

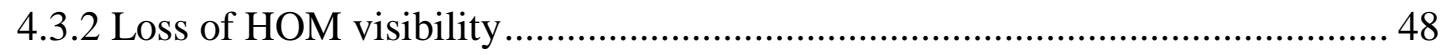

4.3.3 Asymmetric dispersion of wave packet ............................................. 50

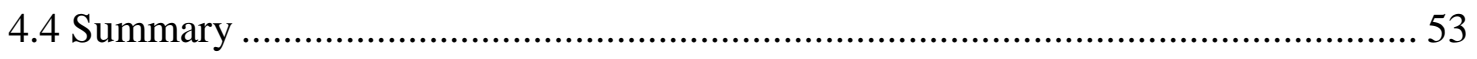

CHAPTER V CONCLUSION AND FUTURE WORK ........................................... 54

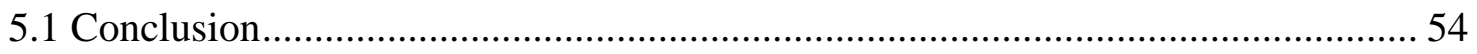

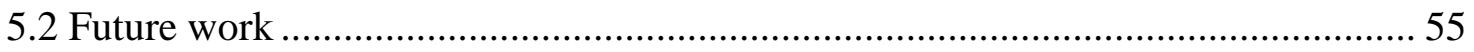

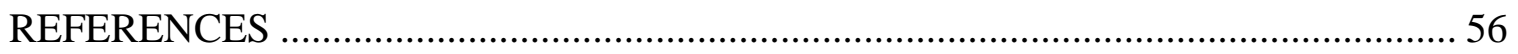

APPENDIX Matlab Code Used in This Work ........................................................... 58 


\section{LIST OF FIGURES}

Figure 2.1: Sketch of the nonlinear mixing process in terms of photon energy.

Figure (a) shows the SFG process Figure (b) shows the DFG process................

Figure 2.2: Type 1 phase matching in lithium niobate for DFG between two inputs

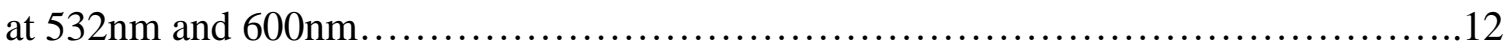

Figure 2.3: The structure shows the function of d-coefficient in terms of periodic

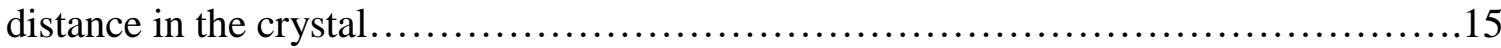

Figure 2.4: The sketch of SPDC process.......................................... 17

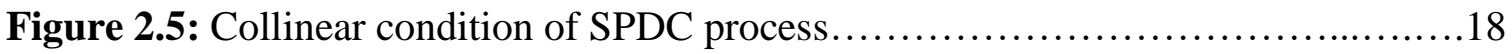

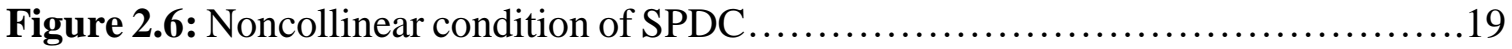

Figure 2.7: Plot periodicity as the function of pump wavelength....................20

Figure 2.8: Phase matching efficiency function, plotted versus pump wavelength $\lambda \ldots \ldots 21$

Figure 2.9: Quasi-phase matching periodicity versus pump wavelength...............21

Figure 2.10 Noncollinear phase matching efficiency, plotted versus pump

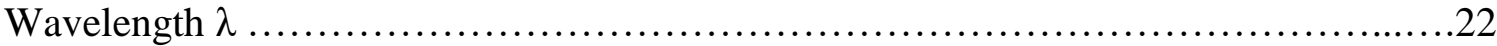

Figure 3.1: Schematic diagram of HOM effect ..................................25

Figure 3.2: Sketch of incident, reflection and transmitted wave at the 50/50 beam

splitter..................................................28

Figure 3.3: Plane wave's reflection and transmittance at beam splitter, plotted versus

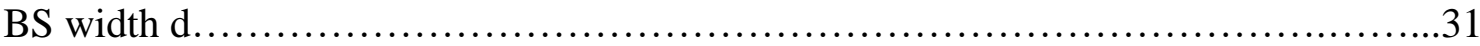

Figure 3.4: The loss exists in the beam splitter when $n 1$ is a complex number...........32

Figure 3.5: Sketch of HOM interferometer.................................... 34

Figure 4.1: Set up sketch of Mandel dip experiment.............................40 
Figure 4.2: Simulation of HOM dip, photon coincidence is plotted versus BS position shift.................................................................... 43

Figure 4.3: HOM dip measured by experiment, aberration bar shows the uncertainty...44

Figure 4.4 Plot idler frequency as the function of signal frequency under the different

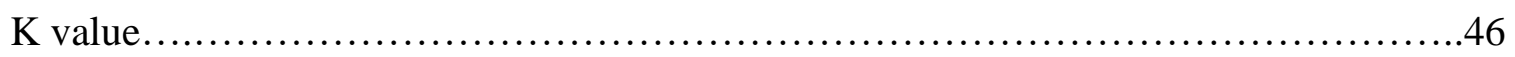

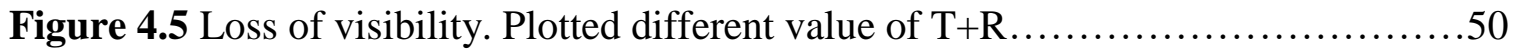

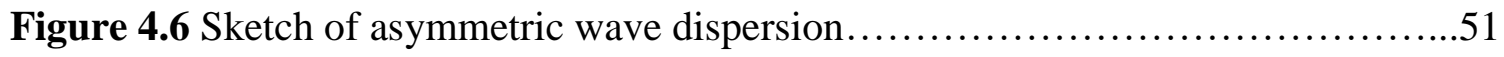

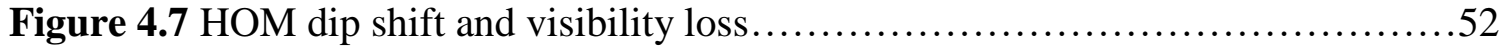




\section{LIST OF ABBREVIATIONS}

PPLN

KDP

$\mathrm{BBO}$

SFG

SHG

DFG

SPDC

HOM
Periodically-poled lithium niobate

Potassium dihydrogen

Beta barium borate

Sum frequency generation

Second harmonic generation

Different frequency generation

Spontaneous parametric down conversion

Hong-Ou-Mandel 


\section{CHAPTER I}

\section{INTRODUCTION}

\subsection{Motivation of this work}

Quantum computing started as a field around 1982, when it was first proposed by Richard Feynman. 1994 is a big year for quantum computing, a famous mathematician Peter Shor proposed a quantum algorithm (an algorithm that runs on a quantum computer) for integer factorization, named as "Shor's algorithm" [1] [2]. It is aimed at creating devices that use the principles of quantum mechanics.

Compared to classical computers available in the present market, quantum devices have an enormous potential, as they provide exponentially faster processing times in certain limits. At the heart of quantum computing is manipulation, characterization, and preservation of quantum properties of various optical and electrical states. Quantum measurement is a very important part of research in the field of quantum information, because it allows the experimental assessment of various components of quantum circuitry, such as a memory, a gate, or a communication channel.

Quantum computing also offers a way to create a new way to think about photons interaction. For example, in a classical treatment, if there are two identical photons input into a beam splitter, there must be 4 possible conditions of the output behaviors: Both of photons are reflected; both of photons are crossly transmitted; one photon is transmitted, the other one is reflected. However, in quantum optics, there are only two possible outcomes due to the quantum interference. Such a phenomenon tells us, that the previous theory of classic field may not be suited in quantum applications. In fact, there are a lot of surprising result in quantum optics which subvert our perception. 
In my research, I will primally focus on the spontaneous parametric down conversion (SPDC) process of the single photon source at the Hong-Ou-Mandel interferometer. In another word, the Hong-Ou-Mandel interferometer is used as a tool to measure the visibility the photon-photon interference. In order to deeply understand the photon correlation and anticorrelation process (photon anticorrelation is also called photon antibunching), we start our work from calculating the phase matching requirement and phase matching bandwidth of the SPDC process in the classic field. Then we consider the photon entanglement and correlation in the quantum field.

We will base our discussion on the analysis of well-known interferometers, such as the event-ready version of the Hong-Ou-Mandel interferometer and the BraunsteinMann Bell state analyzer. We will also analyze the role of distinguishing information in the nonlinear sign-shift gate that constitutes the basic element of the recently proposed scheme for quantum computing based on nonlinear optics.

\subsection{Structure of this work}

Quantum nonlinear optics is a very broad range topic, so we cannot cover everything in detail. Therefore, in this thesis we mainly discuss our topic in the following three aspects:

1. Nonlinear parametric processes and phase matching processes

2. The Hong-Ou-Mandel (HOM) interferometer and single photon sources

3. Mandel dip experiment and photon visibility

In chapter II, we will derive the nonlinear polarization through a field-dependent susceptibility $\chi$, and mainly focus on the second order nonlinear process, $\chi^{(2)}$. Through 
the expression of second order polarization $P^{(2)}$, we discuss multi-waves mixing processes, such as difference frequency generation (DFG), sum frequency generation (SFG) and second harmonic generation (SHG). Then, we give an example of Type I phase matching on the basis of three waves' DFG process. Using lithium niobate as the nonlinear medium of spontaneous parametric down conversion (SPDC), we obtain the expression of phase matching efficiency through calculating the quasi-phase matching requirement and Sellmerier equations.

In chapter III, we introduce the Hong-Ou-Mandel effect, and analyze it by deriving the photon state equation. In order to know how the electric field varies at a 50/50 beam splitter, we assume a plane wave incident on the beam splitter at 45 degree, then observe the properties of the beam splitter by plotting the transmittance and reflection as the function of the beam splitter's width. After doing the preparation work, we introduce the structure of HOM interferometer and how the set up works. To simplify the simulation model for the following chapter, we only consider the source as a perfect single photon source.

In chapter IV, we analyze the Mandel dip experiment on the base of HOM interferometer, and we explain the generation of the HOM dip is due to the displacement of the beam splitter in the interferometer. Meanwhile, we derive the distribution of the observable photons coincidence from the expression of photon phase, and simulate this result via Matlab. On the base of HOM dip result, we give the definition of the photon interference fringe visibilities. In order to explore what factors will influence the photon visibility, we give the criteria for photon pairs' entanglement, then obtain the eigenvalue $\mathrm{K}$ of the Schmidt equation to measure how much entanglement is present in photon pairs. 
At last, plotting the idler frequency as the function of the signal frequency under the different $K$ value to show how is eigen value affects the correlation. 


\section{CHAPTER II}

\section{NONLINEAR PARAMETRIC PROCESS AND PHASE MATCHING}

\subsection{Nonlinear parametric processes}

The polarization induced in the nonlinear medium has a close relation with the susceptibility, $\chi$. Assuming the incident field is the weak electric filed dependence, then, the susceptibility, $\chi$, can be written as a power-series expansion:

$$
\chi=\chi^{(1)}+\chi^{(2)} E(t)+\chi^{(3)} E^{2}(t)+\cdots
$$

Here, $\chi^{(1)}, \chi^{(2)}, \chi^{(3)}, \ldots$ represent the various expansion orders of susceptibility.

Using the expansion in Equation (2.1.1), it's easy to result in a real polarization:

$$
P=\varepsilon_{0} \chi^{(1)} E(t)+\varepsilon_{0} \chi^{(2)} E^{2}(t)+\varepsilon_{0} \chi^{(3)} E^{3}(t)+\cdots
$$

Equation (2.1.2) shows the general nonlinear polarization expansion. In many cases, the nonlinear medium is a $\chi^{(2)}$ medium, such as lithium niobate (PPLN: periodically-poled lithium niobate), potassium dihydrogen (KDP), beta barium borate (BBO) and so on. Therefore, we consider the nonlinear process within a $\chi^{(2)}$ medium as a proper type for this work. Let's represent the total input field as a superposition of the two scaler fields:

$$
E=\left[E_{2} \cos \left(k_{2} z-\omega_{2} t+\phi_{2}\right)+E_{3} \cos \left(k_{3} z-\omega_{3} t+\phi_{3}\right)\right]
$$

Put equation (2.1.3) into the $\chi^{(2)}$ term gives a response which is proportional to the field squared:

$$
P^{(2)}=\varepsilon_{0} \chi^{(2)} E^{2}(t)=\varepsilon_{0} \chi^{(2)}\left[E_{2}^{2} \cos ^{2}\left(k_{2} z-\omega_{2} t+\phi_{2}\right)\right.
$$


$+E_{3}^{2} \cos ^{2}\left(k_{3} z-\omega_{3} t+\phi_{3}\right)+2 E_{2} E_{3} \cos \left(k_{2} z-\omega_{2} t+\phi_{2}\right) \cos \left(k_{3} z-\omega_{3} t+\phi_{3}\right)$

Carefully observing equation (2.1.4), we can split this equation into several specific nonlinear process by expanding it with trigonometric identities [3]:

$$
\begin{gathered}
P^{(2)}=\varepsilon_{0} \chi^{(2) *} \\
\left\{\begin{array}{c}
\frac{1}{2} E_{2}^{2} \cos \left[2\left(k_{2} z-\omega_{2} t+\varphi_{2}\right)\right]+\frac{1}{2} E_{2}^{2} \cos \left[2\left(k_{2} z-\omega_{2} t+\varphi_{2}\right)\right] \quad \text { SHG of } \omega_{2} \text { and } \omega_{3} \\
+E_{2} E_{3} \cos \left[\left(k_{2}+k_{3}\right) z-\left(\omega_{2}+\omega_{3}\right) t+\left(\varphi_{2}+\varphi_{3}\right)\right] \quad \text { SFG of } \omega_{2} \text { and } \omega_{3} \\
+E_{2} E_{3} \cos \left[\left(k_{2}-k_{3}\right) z-\left(\omega_{2}-\omega_{3}\right) t+\left(\varphi_{2}-\varphi_{3}\right)\right] \quad \text { DFG between } \omega_{2} \text { and } \omega_{3}
\end{array}\right.
\end{gathered}
$$

Where SHG means second harmonic generation, SFG means sum frequency generation and DFG means different frequency generation or we can call it "different frequency mixing”.

Sum-frequency generation (SFG) is a second order nonlinear optical process based on the annihilation of two input photons at angular frequencies $\omega_{1}$ and $\omega_{2}$, while, one photon at frequency $\omega_{3}$ is generated. The energy conservation can be expressed as:

$$
\hbar \omega_{3}=\hbar \omega_{1}+\hbar \omega_{2}
$$

SHG is the special case of SFG, in which $\omega_{1}=\omega_{2}$, or $\lambda_{1}=\lambda_{2}$.

DFG is the generation of light with a frequency that is the difference between two other frequencies. Difference-frequency generation is a "parametric process", meaning that the photons satisfy energy conservation, which can be expressed as:

$$
\hbar \omega_{1}-\hbar \omega_{2}=\hbar \omega_{3}
$$

Here is the sketch to show the processes of SFG and DFG [4] 

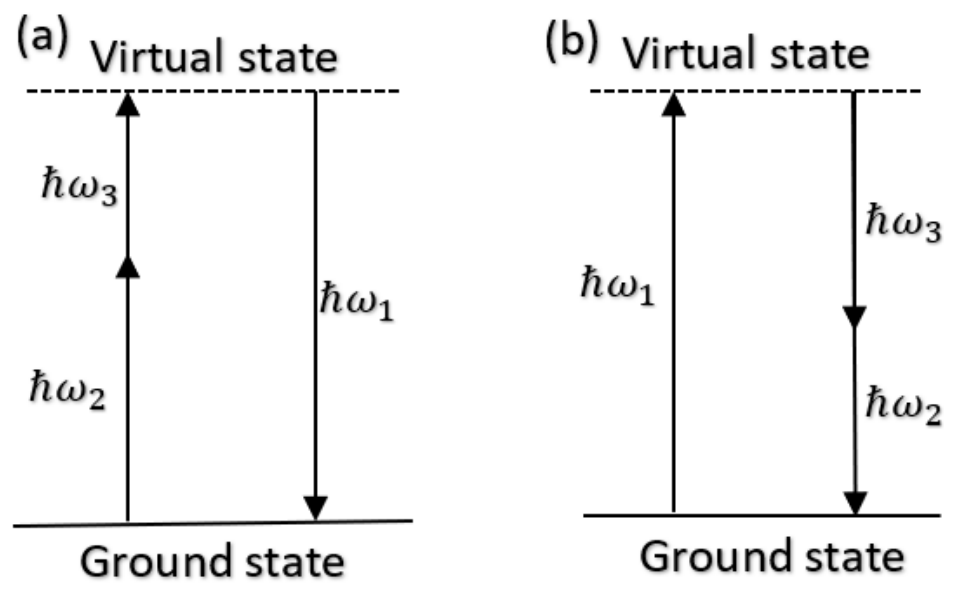

Figure 2.1 Sketch of the nonlinear mixing process in terms of photon energy. Figure (a) shows the SFG process Figure (b) shows the DFG process

Equation (2.1.5) shows two incident waves generating nonlinear polarizations in terms of sum and difference frequency generation process. While a representation of the process in Equation (2.1.5) is shown in figure (2.1).

For most nonlinear $\chi^{(2)}$ processes, the photons do not interact the energy states of the material itself. Therefore, the excited state shown in figure (2.1) is a transient state, or we can call it virtual state.

In section 2.1, we derive the expression of nonlinear polarization from the nonlinear susceptibility, especially focusing on the expression form of the $\chi^{(2)}$ process. After rewriting the second order polarization $P^{(2)}$, we recognize some specific nonlinear mixing process. For example, sum frequency generation and difference frequency generation. In the process of wave mixing, energy conservation is only the basic consideration; besides this, we still need to obtain the requirement of wave phase matching. The birefringent phase matching process will be shown in section 2.2. Also, in 
the next section, we'll show the details of how to find the incident light angle to satisfy the perfect phase matching requirement.

\subsection{Birefringent phase matching}

The three waves mixing process is the most common phase-matched cases in nonlinear optics. As for the $\chi^{(2)}$ process, the second order polarization radiate at the angular frequency $\omega_{3}$ is closely related to the wave vector $k_{3}=\frac{n(\omega) \omega}{c}$. From equation

2.2.2, note that phase matching occurs when $k_{3}=k_{1}-k_{2}$. The more conventional way to convey the phase matching condition for three wave mixing is through the definition:

$$
\Delta k=k_{1}-k_{2}-k_{3}
$$

When $\Delta k=0$, the three waves mixing process is phase matched, and this is the phase matching condition for all three waves interaction process.

In order to more easily analyze the variation of the wave function, we expect the variation of wave field is slowly or occurs gradually over many optical cycles. This assumption is called as slowly varying envelope approximation (SVEA). [5]

In second-order processes, the plane wave equation in the SVEA approximation is:

$$
2 i k \frac{d A}{d z} e^{i k z}=-\omega^{2} \mu_{0} P^{(2)} .
$$

Where $P^{(2)}$ represent the polarization of second order nonlinear process, and it's defined as:

$$
P^{(2)}=2 \varepsilon_{0} d_{e f f} A_{1} A_{2}^{*} e^{i\left(k_{1}-k_{2}\right) z}
$$


While the amplitude $A$ is the wave amplitude. In this work, we only consider three wave mixing process. The wave amplitude and vector amplitude are $A_{1}, A_{2}, A_{3}$ and $k_{1}$, $k_{2}, k_{3}$, respectively.

The first order differential equation for three wave interaction of the amplitude $A_{3}$ is:

$$
2 i k_{3} \frac{d A_{3}}{d z} e^{i k_{3} z}=-\frac{2 \omega_{3}^{2}}{c^{2}} d_{e f f} A_{1} A_{2}^{*} e^{i\left(k_{1}-k_{2}\right) z}
$$

Where $A_{1}, A_{2}$ and $A_{3}$ are the field amplitude. $d_{e f f}$ is the effective nonlinear coefficient, and it's defined as a coefficient for quantifying the strength of a nonlinear interaction. [6]

Generally, the different combinations of polarizations are categorized into two types: Type I and Type II.

Type I phase matching is described as: $o \rightarrow e+e$ (Positive uniaxial).

$$
e \rightarrow o+o \text { (Negative uniaxial). }
$$

Type II phase matching is described as: $o \rightarrow e+o$ or $o \rightarrow o+e$ (Positive uniaxial).

$$
e \rightarrow e+o \text { or } e \rightarrow o+e \text { (Negative uniaxial). }
$$

Where the symbol $o$ represents the ordinary wave, and the symbol $e$ represents the extraordinary wave. Uniaxial crystals are transmissive optical elements in which the refractive index of one crystal axis is different from the other two crystal axes (i.e. $n_{x}=$ $n_{y} \neq n_{z}$ ). Negative uniaxial crystal has the property: $n_{e}<n_{o}$.

When considering a certain three-wave mixing process, the first consideration is which nonlinear medium to use. Let's consider a DFG (different frequency generation) process in lithium niobite between a laser operating at $\lambda_{1}=0.532 \mu \mathrm{m}$, and a second one 
at $\lambda_{2}=0.600 \mu \mathrm{m}$. Lithium niobate is a class $3 \mathrm{~m}$, negative uniaxial crystal that supports Type I and Type II phase matching polarization combinations as given in the last paragraph.

Below is an example to show the relationship between the phase matching angle and the wavelength. Let's assume that Type I phase-matching $e \rightarrow o+o$ of DFG process happens in lithium niobate.

From the energy conservation requirement, DFG process satisfy: $\hbar \omega_{1}-\hbar \omega_{2}=$ $\hbar \omega_{3}$, which can be rewritten as

$$
\frac{1}{\lambda_{1}}-\frac{1}{\lambda_{2}}=\frac{1}{\lambda_{3}}
$$

Therefore, the third operating wavelength should be:

$$
\lambda_{3}=\left(\frac{1}{\lambda_{1}}-\frac{1}{\lambda_{2}}\right)^{-1}=\left(\frac{1}{0.532}-\frac{1}{0.6}\right)^{-1}=4.694 \mu m .
$$

For type I $e \rightarrow o+o$ interaction, the phase difference $\Delta k$ is given by:

$$
\Delta k=2 \pi\left(\frac{n_{e}\left(\lambda_{1}, \theta\right)}{\lambda_{1}}-\frac{n_{o}\left(\lambda_{2}\right)}{\lambda_{2}}-\frac{n_{o}\left(\lambda_{3}\right)}{\lambda_{3}}\right) .
$$

In order to obtain the ordinary and extraordinary refractive index of lithium niobate, we need to know the Sellmeier equation. The Sellmerier equations for lithium niobate phase matching which is found by Edwards and Lawrence in 1984 is shown in Eq. (2.2.5) and Eq. (2.2.6) [7].

$$
n_{o}^{2}=4.9048+\frac{0.11775+2.2314 \times 10^{-8} F}{\lambda^{2}-\left(0.21802-2.9671 \times 10^{-8} F\right)^{2}}+2.1429 \times 10^{-8} F-0.027153 \lambda^{2}(2.2 .5)
$$


$n_{z}^{2}=4.5820+\frac{0.09921+5.2716 \times 10^{-8} F}{\lambda^{2}-\left(0.21090-4.9143 \times 10^{-8} F\right)^{2}}+2.2971 \times 10^{-7} F-0.021940 \lambda^{2}(2.2 .6)$

Where $\lambda$ is entered in micron-meters. F gives the temperature dependence:

$$
F=\left(T-T_{0}\right)\left(T+T_{0}+546\right)
$$

Where $T_{0}=24.5^{\circ} \mathrm{C}$ and $\mathrm{T}$ is entered in ${ }^{\circ} \mathrm{C}$.

In most cases, $T$ is assumed to be room temperature, i.e., $T=T_{0}=24.5 \sim 25^{\circ} \mathrm{C}$

If the ordinary wave is perpendicular to the optical axis, and the extraordinary wave is at the same surface of the optic axis, the refractive of e-wave $n_{e}(\theta)$ is given by:

$$
\frac{1}{n_{e}^{2}(\theta)}=\frac{\cos ^{2}(\theta)}{n_{o}^{2}}+\frac{\sin ^{2}(\theta)}{n_{z}^{2}}
$$

Where the angle $\theta$, is measured with respect to the z-axis (i.e. optic axis). $n_{e}(\theta)$ is the extraordinary index, and it has the explicit dependence on the angle.

Before calculating the e-wave index, we need to calculate the index of o-wave. In this case, assume $T=T_{0}=24.5 \sim 25^{\circ} \mathrm{C}$, thus,

$$
n_{o}\left(\lambda_{2}\right)=\sqrt{4.9048+\frac{0.11775}{0.6^{2}-(0.21802)^{2}}-0.027153 \times 0.6^{2}}=2.2961
$$

Similarly, $n_{o}\left(\lambda_{3}\right)$ can be derived as the same way, $n_{o}\left(\lambda_{3}\right)=2.0765$. Also, $n_{o}\left(\lambda_{1}\right)=$ 2.3232 , and $n_{z}\left(\lambda_{1}\right)=2.2342$. After getting all refractive index value, $\Delta k$ is plotted as a function of $\theta$ and where this curve is equal to zero is the angle of phase matching. Figure $(\mathrm{X})$ shows the plot of $\Delta k$ versus $\theta$ for $e \rightarrow o+o$. 


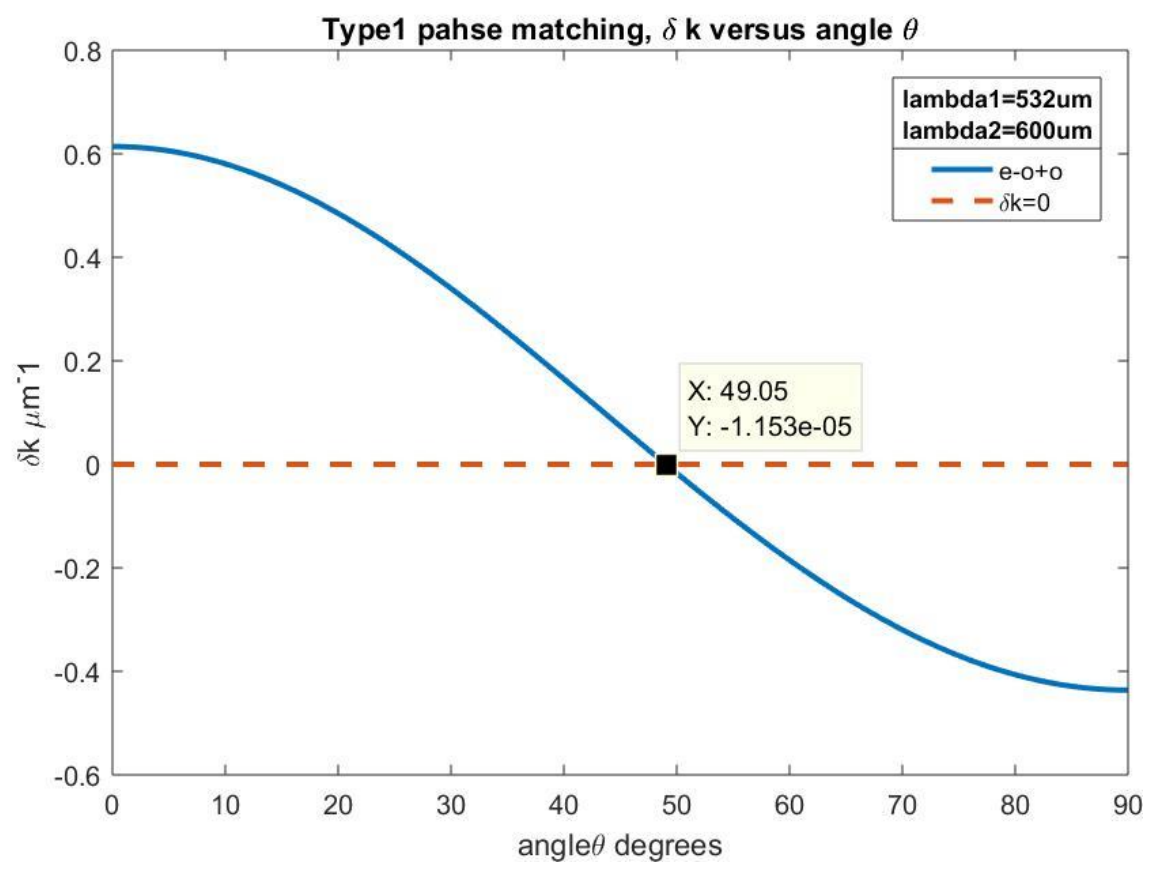

Figure 2.2 Type 1 phase matching in lithium niobate for DFG between two inputs at 532nm and 600nm.

From the figure (2.2), we know the angle where $\Delta k=0$ is the phase-matched angle. And this angle is around 49 degree, which means for the Type I $e \rightarrow o+o$ phase matching, only when the incident two light split at 49.05 degrees, the DFG process of three wave mixing can satisfy the perfect phase matching requirement.

In section 2.2, through solving the SVEA equation, we obtain the phase matching condition of the three waves mixing process. On the base of the phase matching requirement, we further provide two types phase matching conditions in the three waves interaction, then give the Sellmerier equations (lithium niobate) to make sure the refractive index of the signal, idler and output wave. Combine the equation mentioned in section 2.1, we give a specific example of Type I DFG(difference frequency mixing) process to show how to find the phase matching angle and what kind of equation we need to use in the phase matching. 


\subsection{Quasi-phase matching}

From the description of section 2.2, we derive the requirement of phase matching, and also solve for phase matching requirement by using the Sellmeier equation. In this section, we will introduce phase matching techniques which does not require crystal birefringent. This technique is called quasi-phase matching (QPM).

Quasi-phase matching is a technique in nonlinear optics that allows energy to flow from the pump frequency to the positive net of the signal and idler frequency by creating a periodic structure in the nonlinear medium. When the momentum is conserved, as is necessary for phase-matching, through an additional momentum contribution corresponding to the wavevector of the periodic structure. In other words, quasi-phase matching is a technique to provide the additional momentum. [8] Therefore, theoretically, phase matching can be performed for any three-wave mixing process that satisfies energy conservation. For example, all optical frequencies involved can be linear, can have the same polarization, and pass through the media in any direction. This allows us to increase the length of the nonlinear medium and nonlinear interactions.

Quasi-phase matching ensures that positive energy flows from the pump frequency to the signal and idler frequency, even if all the frequencies involved are not locked in each other's phase. When the phase between two light waves is less than 180 degrees, the energy flows from the pump to the signal. If the input two light angle is more than 180 degrees, the energy flows back from the signal to the pump frequency. The coherence length is the length over which the energy flows from the pump to the signal and idler. Each coherence length of the crystal axis is flipped, which allows the energy to continue to flow positively from the pump to the signal and the idle frequency. 
The Quasi-phase matching technique was even proposed before birefringent phase matching, but due to some technical difficulties in fabricating samples, QPM (quasiphase matching) was not as achievable as the phase matching technique at that time. The more direct method of birefringent phase matching was the first to be widely implemented. Both quasi-phase matching and phase matching techniques are now wildly used in variety applications.

In order to understand the properties of QPM, we need to analyze the wave equation. The wave equation for QPM process is the same way as deriving the birefringent phase matching. Let's consider the three-wave mixing process and assume there are two input light and one output light. The intensity of the difference frequency between $\omega_{1}$ and $\omega_{2}$ is obtained using the remaining equation, which can be written as:

$$
\frac{d A_{3}}{d z}=i \frac{\omega_{3}}{n_{3} c} A_{1} A_{2}^{*} d(z) e^{i \Delta k z}
$$

Where $A_{1}, A_{2}$ and $A_{3}$ are the field amplitude.

This equation is directly integrated:

$$
A_{3}=i \frac{\omega_{3}}{n_{3} c} A_{1} A_{2}^{*} \int_{0}^{L} d(z) e^{i \Delta k z} d z
$$

We also need to know the periodic form of the $d(z)$. We begin with a nonlinear crystal that has a periodic structure. Such a periodic structure lends itself naturally to a Fourier series representation, and for the square-wave structure, the series is well known: [9]

$$
d(z)=d_{0} \sum_{m=-\infty}^{\infty} \frac{2}{\pi m} \sin (m \pi D) e^{-i k_{m} z} e^{i \Delta k_{m} z}
$$




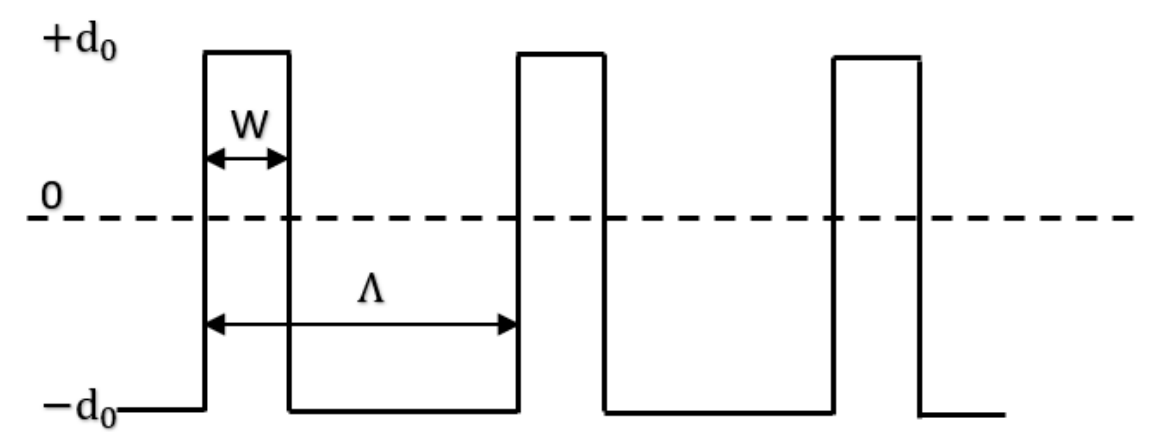

Figure 2.3 the structure shows the function of $d$-coefficient in terms of periodic distance in the crystal

Where $k_{m}$ is given by

$$
k_{m}=\frac{2 \pi}{\Lambda} m
$$

$\mathrm{D}$ is the duty cycle defined by

$$
D=\frac{W}{\Lambda},
$$

where $\mathrm{W}$ is the width of each flips of the periodic crystal and $\Lambda$ is the periodicity.

After a series algebra calculation, the relationship of QPM and birefringent phase matching is expressed as Eq. (2.3.6)

$$
\Delta k_{m}^{\prime}=\Delta k-k_{m}
$$

Substituting Eq. (2.3.4) into Eq. (2.3.6), Eq. (2.3.6) gives:

$$
\Delta k_{m}^{\prime}=\Delta k-\frac{2 \pi}{\Lambda} m
$$

Note that there is only one value of $\mathrm{m}$ when $\Delta k_{m}^{\prime}=0$, all other values of $\mathrm{m}$ yield $\Delta k_{m}^{\prime}$, which can be expressed as periodic function: 


$$
m=m_{0}+k * 2 \pi / \Lambda
$$

The periodicity required for a given quasi-phase matched order is found by letting $\Delta k_{m}^{\prime}=$ 0, therefore, from equation (2.3.7), the periodicity in Eq. (2.3.7) gives:

$$
\Lambda=\frac{2 \pi}{\Delta k} m
$$

Equation (2.3.9) shows the first order of QPM $(\mathrm{m}=1)$ has a shorter periodicity than the higher orders. From the equation (2.3.9), we can also know that a higher order QPM process $(m>1)$ increase the periodicity.

In the section 2.3, we introduce the definition of quasi-phase matching and how we derive the quasi-phase matching requirement from the periodic structure and the nonlinear efficiency. QPM techniques periodically reset the phase of the nonlinear polarization to maintain a coherent buildup of the nonlinear interaction. The most

common condition for rephasing is via the second order nonlinear crystal, $\chi^{(2)}$. We will provide more details and examples about QPM in the next section.

\subsection{Spontaneous parametric down conversion (SPDC)}

The SPDC (spontaneous parametric down conversion) process is very similar to second harmonic generation; it is a nonlinear instant optical process that converts one photon of higher energy (a pump photon), into a pair of photons (a signal photon and an idler photon) of lower energy. But note that since SPDC is a parametric process, all the wave vectors are along the z-axis, thus, the refractive index of both three waves are not angular dependence. Usually, the propagation vector of these photons can be collinear with the pump laser in which the process is called as collinear SPDC. Otherwise, if signal and 
idler photons have a small angle $\theta$ with the pump laser, known as noncollinear SPDC.

Since the process itself does not change the state of the crystal, both energy and momentum must be conserved. As for the three waves mixing process, the energy conservation condition can be written as:

$$
\omega_{P}=\omega_{S}+\omega_{I}
$$

Where $\omega_{P}$ represents the angular frequency of the pump photons, $\omega_{S}$ and $\omega_{P}$ represent the angular frequency of the signal and idler, respectively. The following diagram shows the process of SPDC [10]

\section{Spontaneous Parametric Downconversion}

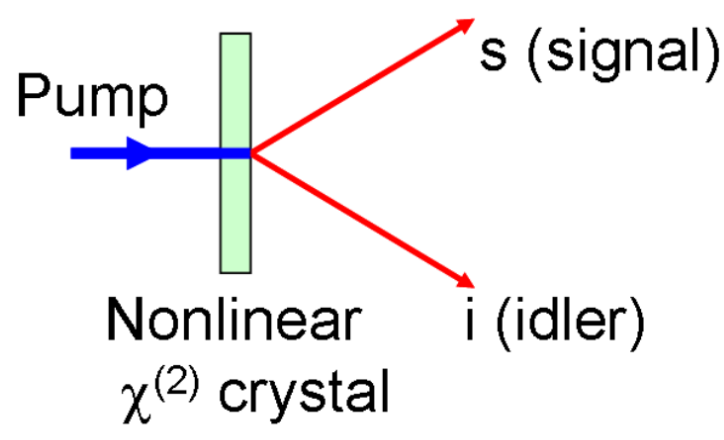

Figure 2.4 The sketch of SPDC process

Usually, there are two types SPDC process, collinear and noncollinear.

(1) Collinear condition:

If the polarization of the pump, signal and idler are all on axis, when the k-vectors are at $\theta=0^{\circ}$ and $90^{\circ}$ to the optic axis of Lithium Niobate. Let choose $\theta=90^{\circ}$ and the walk-off 
is 0 . This is the most ideal configuration, which both pump, signal and idler are polarized in Z-axis (Fig. 2.5).

For the collinear condition, the phase matching condition is the same as the equation given in Eq. (2.2.1). But the refractive index is the index along z-axis $n(\lambda)=n_{z}(\lambda) . \Delta k$ can be written as:

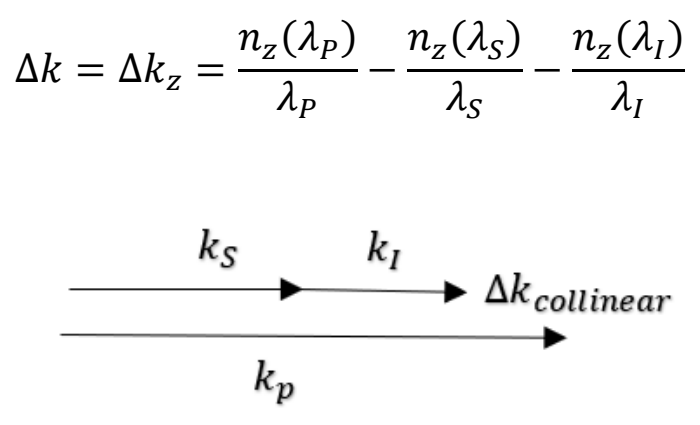

Figure 2.5 collinear condition of SPDC process

(2) Noncollinear interaction

Noncollinear interaction means that signal and idler are not strictly along the Z-axis.

There is a small angle $\psi$ between pump and signal.

The phase matching for the propagation direction (Fig. 2.6) is: (z-axis)

$$
\Delta k_{z}=\Delta k \cos \psi=k_{1}-k_{2} \cos \psi-k_{3} \cos \psi
$$

Then, the phase matching for the orthogonal direction is: (E.g., along the x-axis),

$$
\Delta k_{x}=\Delta k \sin \psi=k_{2} \sin \psi-k_{3} \sin \psi
$$




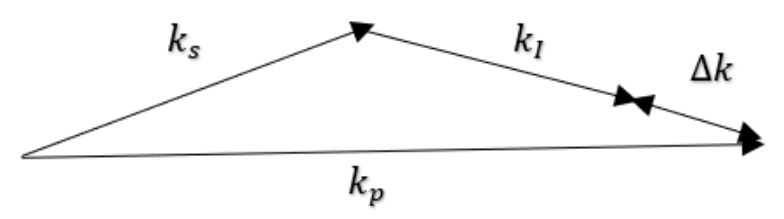

Figure 2.6 noncollinear condition of SPDC

In order to understand the details about the phase matching of SPDC, we'll provide the specific example to show phase matching or quasi-phase matching for both collinear and noncollinear SPDC process. Let's consider the pump and signal wavelength are $0.8 \mu \mathrm{m}$ and $1.6 \mu m$, respectively. For the collinear condition, the three waves cannot phase matched automatically, because the angle of the signal, idler and pump laser are fixed. Thus, we need to use quasi-phase matching condition given in equation (2.3.7) and (2.3.9) to find the periodicity $\Lambda$ to make $\Delta k_{m}^{\prime}=\Delta k-\frac{2 \pi}{\Lambda} m=0$.

Putting the phase matching requirement shown in Eq. (2.3.1) into Eq. (2.3.9), (assume $m=1)$.

we can obtain the expression of the periodicity $\Lambda$ :

$$
\Lambda=\frac{2 \pi}{\Delta k}=\frac{1}{\left(n_{z 1} / \lambda_{1}\right)-\left(n_{z 2} / \lambda_{2}\right)-\left(n_{z 3} / \lambda_{3}\right)}
$$

It's easy to get the idler light's wavelength $(1.6 \mu \mathrm{m})$ from the energy conservation. Using the Sellmeier equation of lithium niobate to calculate the refractive index along z-axis, then regard pump wavelength as a variable, plot the periodicity as the function of pump wavelength to find the largest $\Lambda$. 


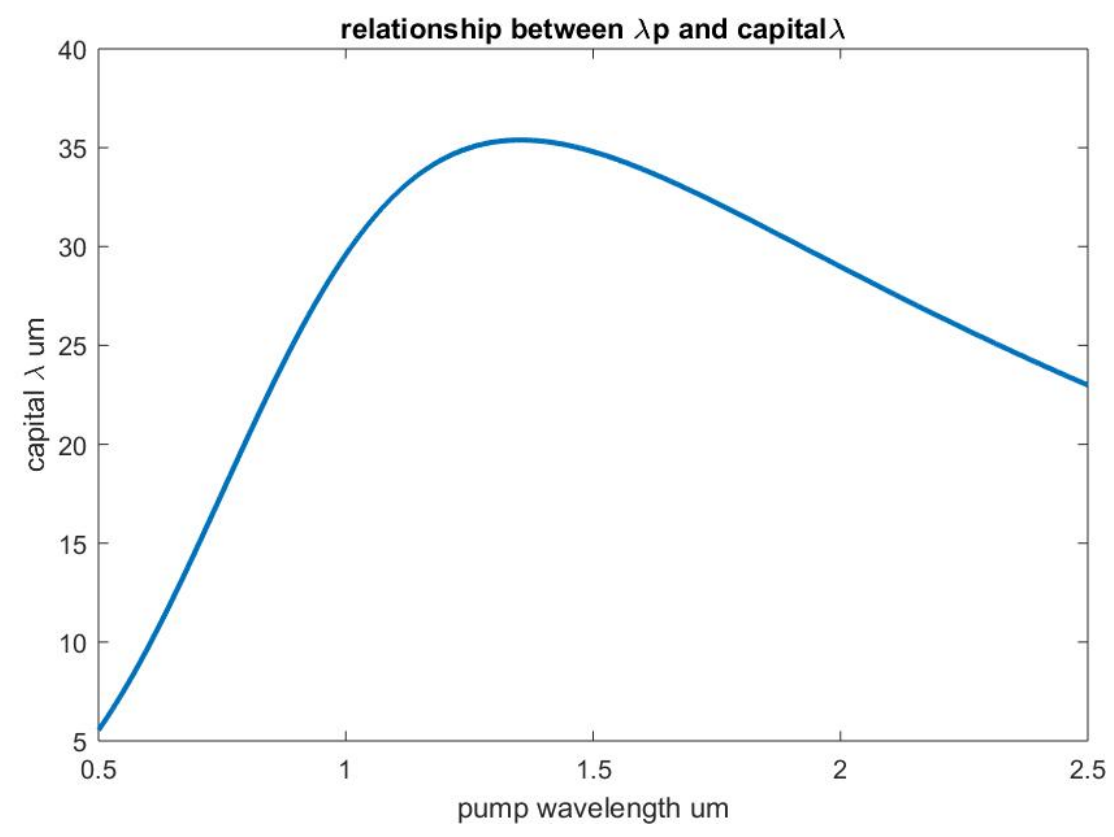

Figure 2.7 plot periodicity as the function of pump wavelength (collinear)

From the figure (2.7), we find the largest periodicity is around $35.39 \mu \mathrm{m}$, corresponding to the minimum $\Delta k$ and the higher phase matching efficiency $\eta$, where the efficiency $\eta$ is given by:

$$
\eta=\frac{I(\Delta k)}{I(\Delta k=0)}=\operatorname{sinc}^{2}\left(\frac{\Delta k L}{2}\right)
$$

From equation (2.4.6), It's clear that the smaller $\Delta k$ is, the lager phase matching efficiency we can get, which is the reason to find the maximum value of periodicity.

Taking the maximum periodicity value in to Equation (2.4.6), and using Sellmerier equation again, we can obtain the phase matching efficiency as the function of pump wavelength, and phase matching bandwidth as well. Figure (2.8) shows the phase matching bandwidth. 


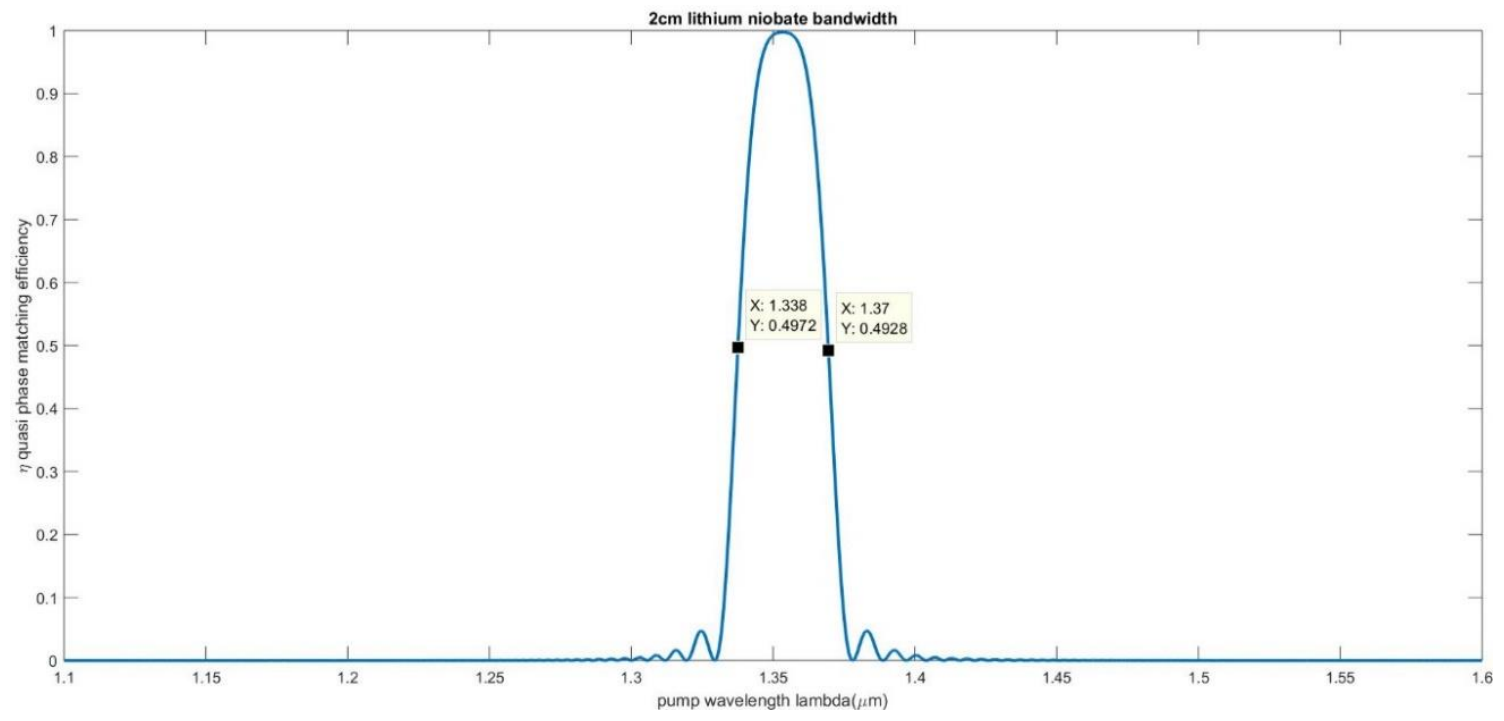

Figure 2.8 phase matching efficiency function, plotted versus pump wavelength $\lambda_{1}$

From the figure (2.8) shown above, the FWHM bandwidth of the phase matching is around 30nm. $\left(F W H M=X_{2}-X_{1}=0.03 u m=30 \mathrm{~nm}\right)$

Similarly, the bandwidth of the noncollinear condition can be calculated and simulated as the same process as the collinear condition. Here are the relationship between periodicity and pump wavelength.

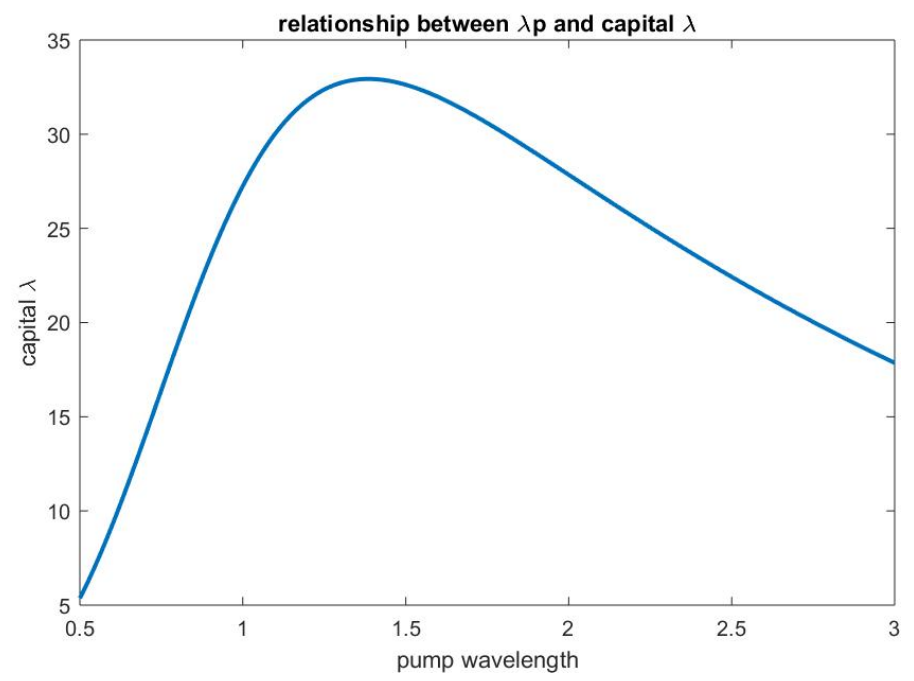

Figure 2.9 quasi-phase matching periodicity versus pump wavelength (noncollinear) 
Figure (2.9) shows the maximum periodicity value to be around $32.93 \mu m$, take this value back into the phase matching efficiency expression. Figure (2.10) shows the phase matching efficiency versus pump wavelength.

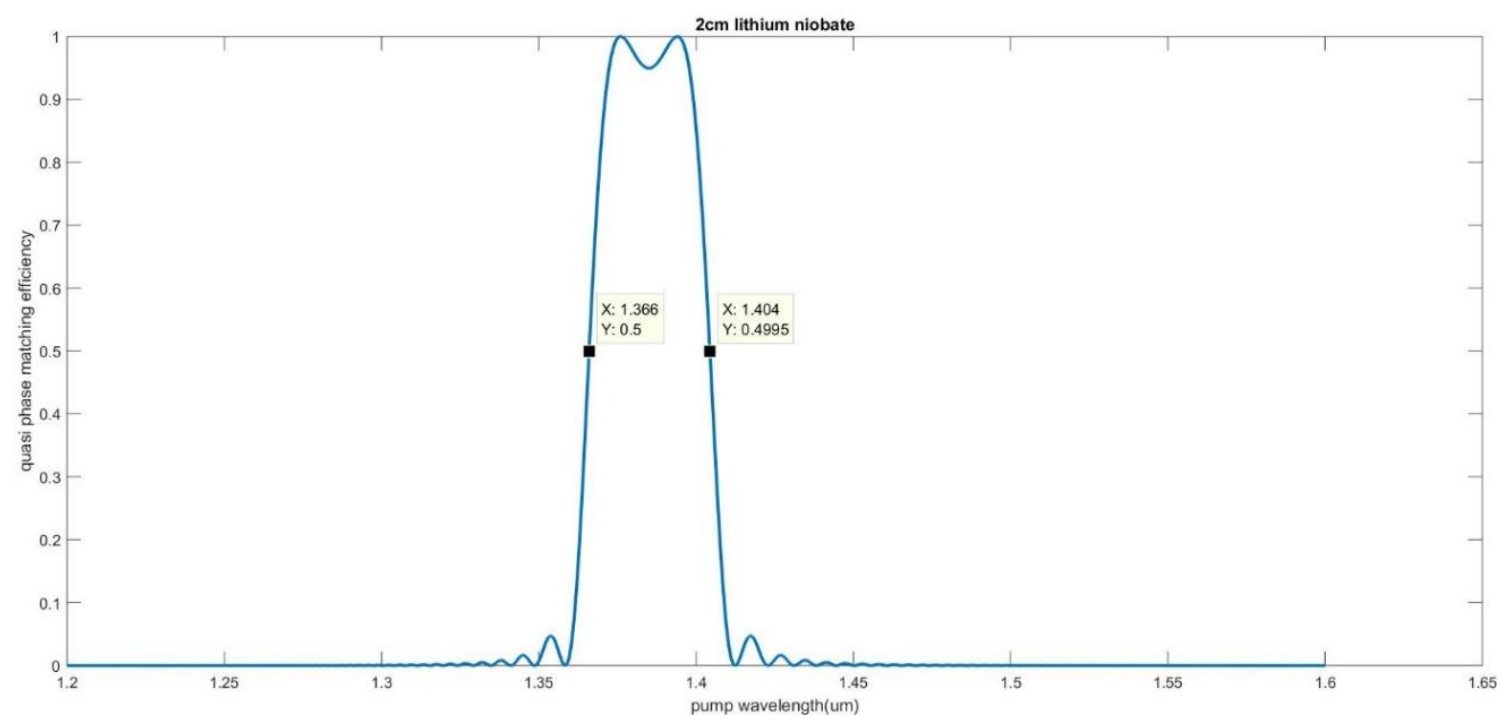

Figure 2.10 noncollinear phase matching efficiency, plotted versus pump wavelength $\lambda_{1}$

From the figure (2.10) shown above, the bandwidth of the noncollinear phase matching is around 38nm. $\left(F W H M=X_{2}-X_{1}=0.038 u m=38 n m\right)$

In this section, we introduce two types of SPDC process: collinear and noncollinear. Combining the quasi-phase matching background, we give a certain example to show how to get the phase matching bandwidth and efficiency of these two types of SPDC process. Note that SPDC is not a true single photon source since the photons are not antibunched. However, when one of the photons is heralded, it then exhibits single photon characteristics.

In the quantum mechanics, the photons with identical spectra were selected to use filtering and single-mode coupling, more details will be provided in Chapter III. 


\subsection{Summary}

In this chapter, we introduced the nonlinear polarization through a field-dependent susceptibility. We derived this connection by using a classical picture based on the harmonic oscillator picture. Also, on the process of deriving three wave mixing, we give the requirement of phase matching and provide a specific example to show the relationship of phase matching condition and incident wave vector's angle. We notice that the phase matching is not applicable when the gain medium has periodic structure, so we proposed the idea of quasi-phase matching.

At the same time, this chapter, as the foundation of the Hong-Ou-Mandel effect discussed in chapter III and IV, explains the SPDC generation process in detail. And we compute the phase matching bandwidth of photon mixing process through giving a Type I DFG nonlinear process.

In this thesis, we restrict our discussion by primarily focusing on the nonlinear susceptibility of $\chi^{(2)}$, which is the origin of the second order nonlinear effect. 


\section{CHAPTER III}

\section{HOM INTERFEROMETER AND SINGLE PHOTON SOURCES}

\subsection{Hong-Ou-Mandel effect}

A HOM (Hong-Ou-Mandel) interferometer is based on a second-order coherence effect between two single photon fields that are incident onto a 50/50 beam splitter (50/50 beam splitter means both of transmittance and reflection are equal to $50 \%, T=R=50 \%$ ). The interferometer is based on the famous effect which is called Hong-Ou-Mandel effect that the two indistinguishable photons entangle and correlate with each other at the beam splitter surface, in such a way that the two photons would never leave out the beam splitter via the separate output ports. [11] In other words, the input photons will entangle with each other and will leave the beam splitter only at the same side.

When a photon enters a beam splitter, there are two possibilities: it will either be reflected or transmitted. The relative probabilities of transmission and reflection are determined by the reflectivity of the beam splitter. Here, we assume a 50/50 beam splitter, in which a photon has equal probability of being reflected and transmitted.

Consider two identical photons intersect into a 50/50 beam splitter, there are 4 possible conditions of the output behaviors:

1. Both of photons are reflected into the same side where they incidence.

2. Both of photons are crossly transmitted into the other side.

3. The above photon is transmitted, the below photon is reflected.

4. The above photon is reflected, the below photon is transmitted. [12] 


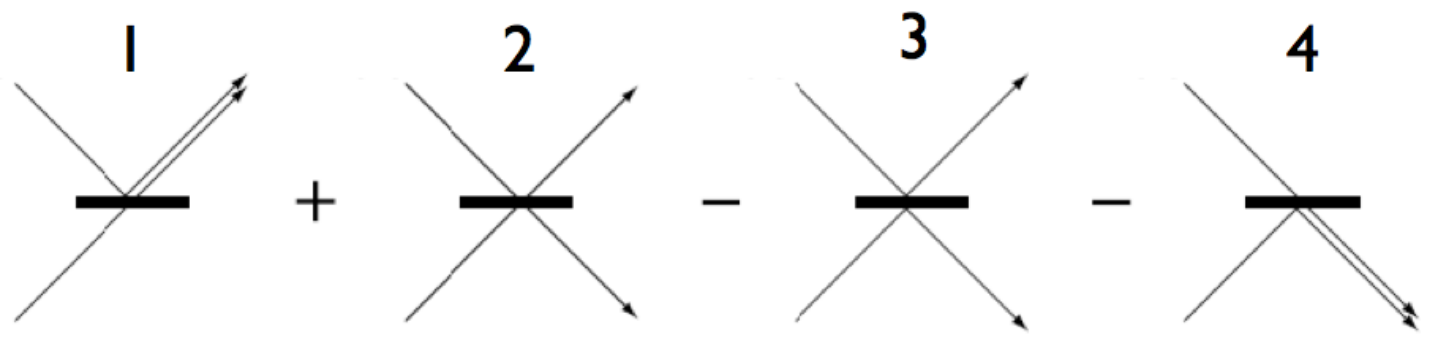

Figure 3.1 schematic diagram of HOM effect [12]

Assume both of two photons have the same physical properties and incident with 45 degree.

Assuming both of two incident photons are at the ground and single mode state. $\widehat{a_{1}^{\dagger}}, \widehat{a_{2}^{\dagger}}$ are the input single photon state, while $\widehat{a_{3}^{\dagger}}, \widehat{a_{4}^{\dagger}}$ are the output photon states.

$$
\begin{gathered}
\widehat{a_{1}^{\dagger}} \widehat{a_{2}^{\dagger}}|0,0\rangle_{a 1 a 2}=|1,1\rangle_{a 1 a 2} \\
\widehat{a_{3}^{\dagger}}=t \widehat{a_{1}^{\dagger}}+r \widehat{a_{2}^{\dagger}} \\
\widehat{a_{4}^{\dagger}}=r \widehat{a_{1}^{\dagger}}+t \widehat{a_{2}^{\dagger}}
\end{gathered}
$$

Because the beam splitter satisfies $\mathrm{T}=\mathrm{R}=50 \%$, (where $T=|t|^{2}, R=|r|^{2}$ ), then, the amplitude reflection and transmission $\mathrm{t}$ and $\mathrm{r}$ must satisfy $t=r^{*}$, which means $t^{2}=-r^{2}$ Thus, we have,

$$
\widehat{a_{3}^{\dagger}} \widehat{a_{4}^{\dagger}}=\operatorname{tr}\left({\widehat{a_{1}^{\dagger}}}^{2}+{\widehat{a_{2}^{\dagger}}}^{2}\right)+\left(r^{2}+t^{2}\right) \widehat{a_{1}^{\dagger}} \widehat{a_{2}^{\dagger}}=\operatorname{tr}\left({\widehat{a_{1}^{\dagger}}}^{2}+{\widehat{a_{2}^{\dagger}}}^{2}\right)
$$

From the equation shown above, the combination terms of $\widehat{a_{1}^{\dagger}} \widehat{a_{2}^{\dagger}}$ vanishes, the only terms left are ${\widehat{a_{1}^{\dagger}}}^{2}$ and ${\widehat{a_{2}^{\dagger}}}^{2}$. This phenomenon means no coincidence should be recorded 
simultaneously by the output port. (More details and derivation of the coincidence will be shown in chapter 4) Let's verify our assumption by inversely input the photons,

$$
t^{*} \widehat{a_{3}^{\dagger}}+r^{*} \widehat{a_{4}^{\dagger}}=\left(|t|^{2}+|r|^{2}\right) \widehat{a_{1}^{\dagger}}+\left(t^{*} r+r^{*} t\right) \widehat{a_{2}^{\dagger}}=\widehat{a_{1}^{\dagger}}+\left(t^{r}+r^{2}\right) \widehat{a_{2}^{\dagger}}=\widehat{a_{1}^{\dagger}}
$$

Similarly, we have:

$$
r^{*} \widehat{a_{3}^{\dagger}}+t^{*} \widehat{a_{4}^{\dagger}}=\left(|t|^{2}+|r|^{2}\right) \widehat{a_{2}^{\dagger}}+\left(t^{*} r+r^{*} t\right) \widehat{a_{1}^{\dagger}}=\widehat{a_{2}^{\dagger}}+\left(t^{r}+r^{2}\right) \widehat{a_{1}^{\dagger}}=\widehat{a_{2}^{\dagger}}
$$

Equation (3.1.5) and (3.1.6) tell us that no matter the direction we choose to inject the single photon pairs, the output photons can only export at the one side of the beam splitter. And these two equations prove our guess that there is no coincidence between two photons recorded by each side detector. (Assume we install the detector $D_{1}$ and $D_{2}$ at the two sides of the beam splitter.)

Conclude the derivation we make, the HOM effect can be easily displayed by the following derivation.

The identical incident photons can be described as:

$$
\widehat{a_{1}^{\dagger}} \widehat{a_{2}^{\dagger}}|0,0\rangle_{a 1 a 2}=|1,1\rangle_{a 1 a 2}
$$

where $|0\rangle$ is vacuum state and $|1\rangle$ is a single ground photon state. When two photons meet at a beam splitter at the same time (There is no phase delay between each input path), which is a unitary transformation, after they interact in the beam splitter, they'll create output states $\widehat{a_{3}^{\dagger}}$ and $\widehat{a_{4}^{\dagger}}$ that can be described as:

$$
\widehat{a_{3}^{\dagger}}=\frac{\widehat{a_{1}^{\dagger}}+\widehat{a_{2}^{\dagger}}}{\sqrt{2}}
$$




$$
\widehat{a_{4}^{\dagger}}=\frac{\widehat{a_{1}^{\dagger}}-\widehat{a_{2}^{\dagger}}}{\sqrt{2}}
$$

When two identical single photons are incident on a beam splitter, their state can be written as

$$
\begin{gathered}
|1,1\rangle_{a 1 a 2}=\widehat{a_{1}^{\dagger}} \widehat{a_{2}^{\dagger}}|0,0\rangle_{a 1 a 2}=\left(\widehat{\frac{a_{3}^{\dagger}}{\sqrt{2}} \widehat{a_{4}^{\dagger}}}\right)\left(\frac{\widehat{a_{3}^{\dagger}}-\widehat{a_{4}^{\dagger}}}{\sqrt{2}}\right)|0,0\rangle_{a 3 a 4} \\
=\frac{1}{2}\left(\widehat{a_{3}^{\dagger}} \widehat{a_{3}^{\dagger}}-\widehat{a_{4}^{\dagger}} \widehat{a_{4}^{\dagger}}\right)|0,0\rangle_{a 3 a 4}=\frac{|2,0\rangle_{a 3 a 4}-|0,2\rangle_{a 3 a 4}}{\sqrt{2}}
\end{gathered}
$$

Therefore, the single photon state $|1,1\rangle_{a 3 a 4}$ vanishes, giving zero probability of the two identical photons being detected simultaneously at different output ports of the beam splitter.

Single photon avalanche detectors produce an electrical pulse each time an incoming photon produces an avalanche. When the two detectors simultaneously detect one photon each, a coincidence count is recorded. If just one of the detectors clicks, no coincidence counts are recorded.

In this section, we introduce the phenomenon of Hong-Ou-Mandel effect, which is a phenomenon of quantum entanglement, and verify the hypothesis of quantum entanglement from the expression derivation of ground state photons. In this section, we have mentioned the distinguishability and the coincidence several times, the more details about the photon coincidence and distinguishability will be introduced in chapter IV. 


\subsection{Electric field at 50/50 beam splitter}

The 50/50 beam splitter is the most common beam splitter for single photon interference. In this section, only consider the photon reflection and transmittance in the classic field, ignore the photon antibunching phenomenon (photon antibunching will be explained in the section 3.3. chapter IV will also contain some photons' correlation and anticorrelation.) The symbol "50/50" represents that both of transmittance and reflection are equal to $50 \%$. $(T=R=50 \%)$. Let's consider the plane wave incident to the beam splitter at 45 degree. Figure (3.2) shows the sketch of the incident wave, transmitted wave and reflection wave.

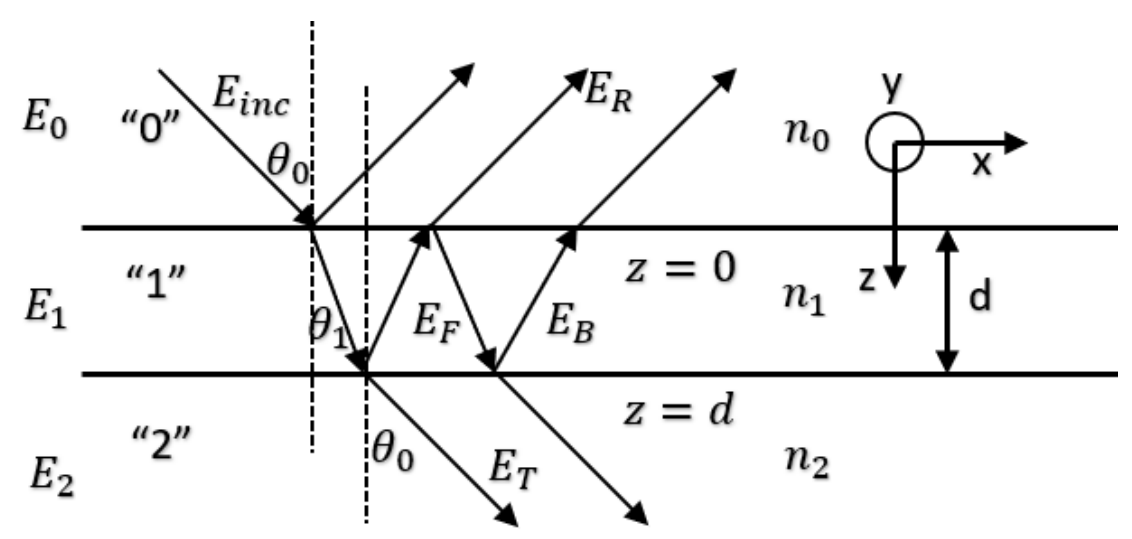

Figure 3.2 sketch of incident, reflection and transmitted wave at the 50/50 beam splitter

Where $\mathrm{d}$ is the width of the beam splitter, $E_{i n c}, E_{R}$ and $E_{T}$ respectively represent the incident wave, reflective wave and transmitted wave. While $E_{F}$ and $E_{B}$ represent the forward and backward reflection wave within the beam splitter, which are the transit parameter, and they would not display at the final expression. $E_{0}, E_{1}$ and $E_{2}$ are the gross electric field of the medium " 0 ", "1" and "2". 
Assume $n_{0}=n_{2}=1.5, n_{1}=2.2+i * a$. Where $a$ is random number, and it can be any value. (the index $n_{1}$ is the complex number, maybe not the pure real number).

Meanwhile, assume $\theta_{0}=\theta_{2}=45^{\circ}$, according to the Snell's law $\left(n_{0} \sin \theta_{0}=n_{1} \sin \theta_{1}\right)$, $\theta_{1}$ can be easily calculated, which is equal to $\theta_{1}=28.824^{\circ}$. Consider the incident light wavelength is $\lambda_{0}=0.8 \mu m$.

From the figure (3.2) shows, the expression of $E_{0}, E_{1}$ and $E_{2}$ gives:

$$
\begin{gathered}
E_{0}=\left(E_{i n c} e^{i k_{z 0} z}+E_{R} e^{-i k_{z 0} z}\right) e^{i k_{x 0} x} \\
E_{1}=\left(E_{F} e^{i k_{z 1} z}+E_{B} e^{-i k_{z 1} z}\right) e^{i k_{x 1} x} \\
E_{2}=E_{T} e^{i k_{z 2} z} e^{i k_{x 2} x}
\end{gathered}
$$

Where $k_{z}$ is the $\mathrm{z}$ axis component of the wave propagation vector $\mathrm{k}$, while $k_{x}$ is the $\mathrm{x}$ axis component of the wave propagation vector $k$.

The wave propagation vector $\mathrm{k}$ can be expressed as:

$$
\begin{gathered}
k_{0}=n_{0} \frac{\omega}{c}=\frac{2 \pi n_{0}}{\lambda_{0}}=k_{2} \\
k_{1}=n_{1} \frac{\omega}{c}=\frac{2 \pi n_{1}}{\lambda_{0}} \\
\left\{\begin{array}{l}
k_{z 0}=k_{0} \cos \theta_{0}=k_{z 2} \\
k_{x 0}=k_{0} \sin \theta_{0}=k_{x 2}
\end{array}\right. \\
\left\{\begin{array}{l}
k_{z 1}=k_{1} \cos \theta_{1} \\
k_{x 1}=k_{1} \sin \theta_{1}
\end{array}\right.
\end{gathered}
$$

Where $\cos \theta_{1}$ and $\sin \theta_{1}$ are given from the Snell's law: 


$$
\begin{gathered}
\sin \theta_{1}=\frac{n_{0}}{n_{1}} \sin \theta_{0} \\
\cos \theta_{1}=\sqrt{1-\sin ^{2} \theta_{1}}=\frac{\sqrt{n_{1}^{2}-n_{0}^{2} \sin ^{2} \theta_{0}}}{n_{1}}
\end{gathered}
$$

In order to obtain the expression of $E_{R}, E_{T}$ in terms of $E_{\text {inc }}$ and $k_{z}$, we still need the boundary conditions, which gives:

$$
\begin{gathered}
\text { when } z=0,\left\{\begin{array}{c}
E_{0}=E_{1} \\
\frac{d E_{0}}{d z}=\frac{d E_{1}}{d z}
\end{array}\right. \\
\text { where } z=d,\left\{\begin{array}{c}
E_{1}=E_{2} \\
\frac{d E_{1}}{d z}=\frac{d E_{2}}{d z}
\end{array}\right.
\end{gathered}
$$

After the complicated algebra calculation, $E_{R}$ and $E_{T}$ can be re-written as:

$$
\begin{gathered}
E_{R}=\frac{\left(1-e^{-2 i k_{z 1} d}\right) E_{i n c}}{\frac{k_{z 1}-k_{z 0}}{k_{z 1}+k_{z 0}} e^{-2 i k_{z 1} d}-\frac{k_{z 1}+k_{z 0}}{k_{z 1}-k_{z 0}}} \\
E_{T} e^{i k_{z 0} d}=\frac{4 k_{z 1} k_{z 1} E_{i n c}}{\left(k_{z 1}+k_{z 0}\right) e^{-i k_{z 1} d}-\left(k_{z 1}-k_{z 0}\right) e^{i k_{z 1} d}}
\end{gathered}
$$

The transmitted amplitude is always combined with the exponential phase term, therefore, we can regard the amplitude transmittance as:

$$
E_{T}^{\prime}=E_{T} e^{i k_{z 0} d}=\frac{4 k_{z 1} k_{z 1} E_{i n c}}{\left(k_{z 1}+k_{z 0}\right) e^{-i k_{z 1} d}-\left(k_{z 1}-k_{z 0}\right) e^{i k_{z 1} d}}
$$

Also, we define the intensity reflection and transmittance as:

$$
R=\left|\frac{E_{R}}{E_{\text {inc }}}\right|^{2}
$$




$$
T=\left|\frac{E_{T}{ }^{\prime}}{E_{\text {inc }}}\right|^{2}
$$

Figure (3.3) shows the relationship between the wave intensity reflection and transmittance, which is plotted as the function of the beam splitter width $\mathrm{d}$.
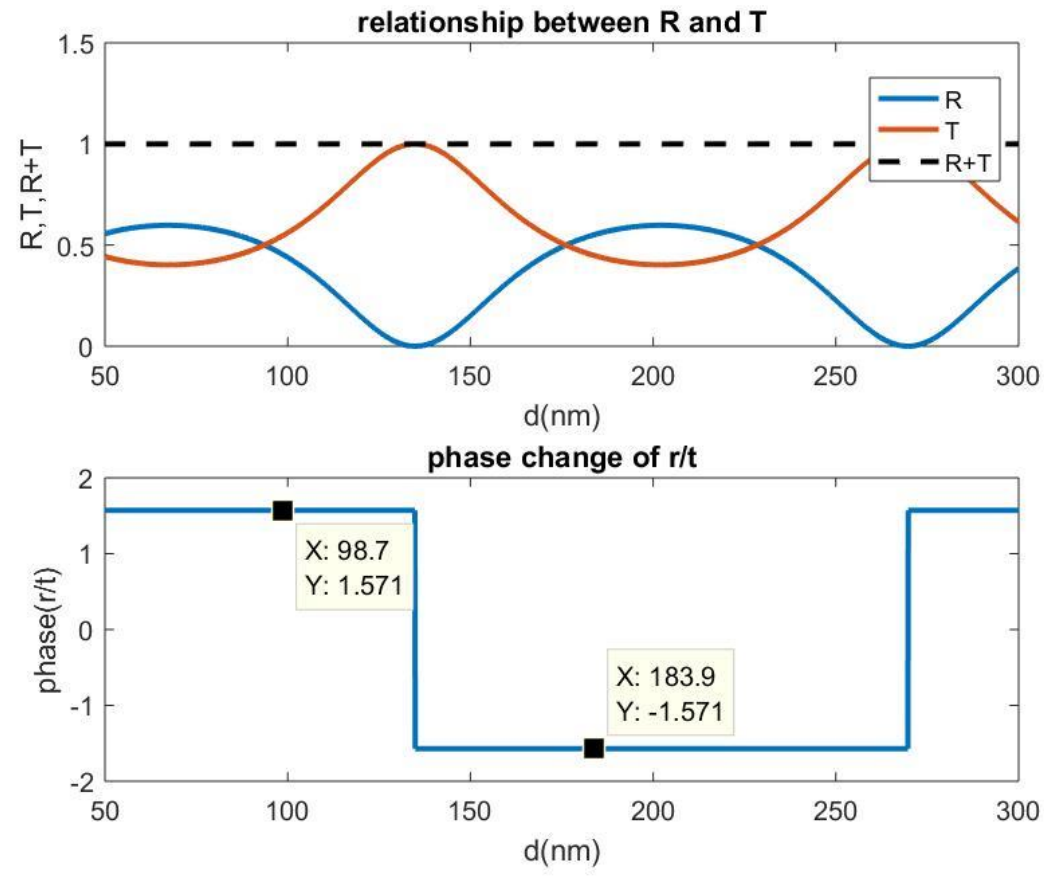

Figure 3.3 plane wave's reflection and transmittance at beam splitter, plotted versus BS width d

In order to prove that in the $50 / 50$ beam splitter, the transmittance of amplitude is the conjugate of the reflection of amplitude $\left(r^{*}=t,|T|=|R|\right)$, we also plot the phase change of $r / t$. It's clear that the phase change from the positive $\frac{\pi}{2}$ to the negative $-\frac{\pi}{2}$. This result is the same as what we mentioned in section (3.1).

When the refractive index of the beam splitter is the complex number, (E.g. $n_{1}=2.2+$ $0.2 i$ ). The loss will be presented in the beam splitter. That means the gain medium may 
absorb some energy from the incident wave. (See the figure 3.4). That's the reason why $T+R<1$.
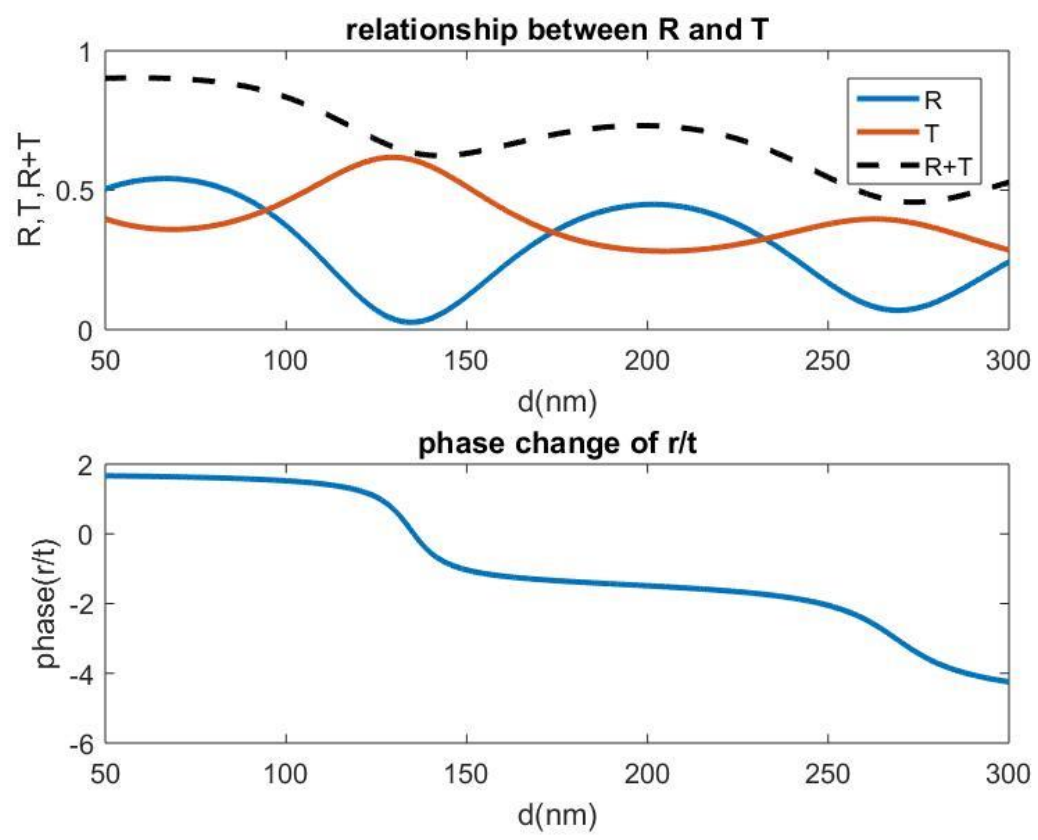

Figure 3.4 the loss exists in the beam splitter when $n 1$ is a complex number

In this section, through a series algebra calculation and derivation, we derive the intensity transmittance and reflection of 50/50 beam splitter, while assuming the incident wave is plane wave and the incident angle is 45 degree.

\subsection{HOM interferometer}

The illustration of HOM interferometer is shown in the figure (3.5) [4] [7], $D_{1}$ and $D_{2}$ are the detectors to count the export photon number. The HOM interferometer is usually used to show the correlation of the photon pairs from the SPDC process. Further speaking, degenerate and distinguishable signal and idler photons are filtered to limit their spectral bandwidth from the Type I SPDC source. 
The HOM interferometer is consisted of the following three parts:

\section{(1). Degeneracy part.}

(2). Intensity correlation part.

(3). Coincidence counting part.

At first, a pump source input into the $\chi^{(2)}$ crystal and generate the degenerate process (SPDC event). Then, though SPDC event, the signal and idler photons meet at the beam splitter surface to do the correlation with each other. Finally, the detector will record and count the output photons from each exit port.

Note that only one detector would detect the photons if the signal and idler photon pairs meet at the beam splitter surface at the same time with the same phase. Under this condition, the coincidence of simultaneously detecting the photons from both $D_{1}$ and $D_{2}$ is zero, which is correspond to the result in section (3.1). However, if add a phase retardation or time retardation at one path of the interferometer, the detectors have some probability to detect the photons from the different ports which means the coincidence of the photon pairs is not zero. Usually, it's not easy to add the phase modulator or retarder to delay the photons' arriving time, so we can slowly shift the beam splitter in $x$ direction to make sure the photon pairs cannot arrive at the beam splitter at the same time. (Define the pump vector is in $z$ direction, $x$ direction is within the paper plane and y direction is perpendicular to the paper plane.)

If we plot the photons' coincidence distribution while slowly moving the beam splitter upward and downward, we can clearly see the dip of the figure. More experimental and 
theoretical details will be showed in the chapter IV, section (4.1). (Mandel dip experiment).

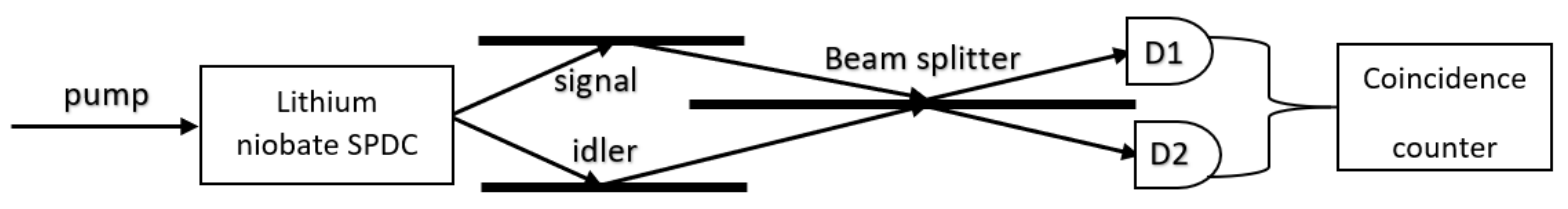

Figure 3.5 sketch of HOM interferometer

\subsection{Degree of coherence and photon antibunching}

\subsubsection{Degree of coherence}

First-order coherence is what you would measure if you shone light into a Michelson interferometer and observe interference fringes. Essentially this is a single-photon interference experiment - interference between the probability amplitudes of a photon taking a particular route through the interferometer. It is a measure of correlations between field amplitudes and it is second order in the field amplitudes.

The first order correlation function can be written as

$$
g^{(1)}(\tau)=\frac{\left\langle E^{*}(t) E(t+\tau)\right\rangle}{\left\langle|E(t)|^{2}\right\rangle}
$$

where the symbol $\langle\ldots\rangle$ denotes the time average of $E(t)$. The first correlation function represents the correlation of the wave amplitude.

Generally, the first correlation function has the following properties:

$$
g^{(1)}(0)=1
$$




$$
g^{(1)}(\tau)=g^{(1)}(-\tau)^{*}
$$

If the input light only has the single frequency, then, $g^{(1)}(\tau)$ can be written as:

$$
g^{(1)}(\tau)=e^{-i \omega_{0} \tau}
$$

For the homogeneous broadening light, such as Lorentzian broadening light,

$$
g^{(1)}(\tau)=e^{-i \omega_{0} \tau-\left(\frac{|\tau|}{\tau_{c}}\right)}
$$

For the inhomogeneous broadening light, such as Doppler broadening light,

$$
g^{(1)}(\tau)=e^{-i \omega_{0} \tau-\frac{\pi}{2}\left(\frac{\tau}{\tau_{c}}\right)^{2}}
$$

Where $\omega_{0}$ is the central frequency of the light and $\tau_{c}$ is the coherence time of the light.

In optical interferometers such as the Michelson interferometer, one splits an electric field into two parts, introduces a time delay to one of the components, and then recombines them. The intensity of resulting field is measured as a function of the time delay. This is used to measure $g^{(1)}(\tau)$ function.

Second order coherence basically describes correlations between field intensities between two points. It is fourth order in field amplitudes. A common test of how good a single photon source is to measure the second-order correlation function. This is basically achieved by firing photons at a beam splitter and placing a detector equidistant from each output port of a 50:50 beam splitter. If it's a perfect single photon source, you will never measure simultaneous detection events.

In the classic physical field, the second order correlation function is expressed as (Assume the wave is the parallel plane wave) 


$$
g^{(2)}(\tau)=\frac{\langle I(t) I(t+\tau)\rangle}{\left\langle|I(t)|^{2}\right\rangle}
$$

Where $I(t)=E(t) E^{*}(t)$. In the electric field, usually we use the expression $g^{(2)}(\tau)$ in terms of intensities, which represent the correlation of the intensities.

Generally, the second order correlation function has the following properties:

$$
\begin{gathered}
g^{(2)}(\tau)=g^{(2)}(-\tau) \\
g^{(2)}(0) \geq 1 \\
g^{(2)}(0) \leq g^{(2)}(\tau)
\end{gathered}
$$

There is a special case for the second order correlation function, when the interferometer is symmetric and the coherent state is 0.5 , each output of the correlation function will be $1\left(g^{(2)}(\tau)=1\right)$.

If the input light is the single frequency light, the coherence of the two bunches of input light is always constant, so the second coherence function can be written as: $g^{(2)}(\tau)=1$.

However, in quantum mechanics, the properties of the coherence function we mentioned in this section are not always satisfied. In the next section, we'll concentrate the photon antibunching process and see why the properties in the classic field are not satisfied for the quantum field.

\subsubsection{Photon antibunching}

Photon antibunching generally refers to a light field with photons more equally spaced than a coherent laser field, a signature being signals at appropriate detectors which are anticorrelated 
The variance of the photon number distribution can be expressed as

$$
V_{n}=\left\langle\Delta n^{2}\right\rangle=\left\langle n^{2}\right\rangle-\langle n\rangle^{2}=\left\langle\left(\hat{a}^{\dagger} \hat{a}\right)^{2}\right\rangle-\left\langle\hat{a}^{\dagger} \hat{a}\right\rangle^{2}
$$

Using commutation relations, Eq.(X) can be written as

$$
V_{n}=\left\langle\left(\hat{a}^{\dagger}\right)^{2} \hat{a}^{2}\right\rangle-\left\langle\hat{a}^{\dagger} \hat{a}\right\rangle-\left\langle\hat{a}^{\dagger} \hat{a}\right\rangle^{2}
$$

Because the photon state $\langle n\rangle$ can be expressed as $\langle n\rangle=\left\langle\hat{a}^{\dagger} \hat{a}\right\rangle$, we take this expression back to Eq.(X) and obtain:

$$
V_{n}-\langle n\rangle=\left\langle\left(\hat{a}^{\dagger}\right)^{2} \hat{a}^{2}\right\rangle-\left\langle\hat{a}^{\dagger} \hat{a}\right\rangle^{2}
$$

In quantum mechanics, the second order correlation no longer represents the correlation of the wave intensity, the correlation function is written in terms of the creation $\hat{a}$ and annihilation $\hat{a}^{\dagger}$, which can be expressed as:

$$
g^{(2)}(\tau)=\frac{\left\langle\hat{a}^{\dagger}(t) \hat{a}^{\dagger}(t+\tau) \hat{a}(t+\tau) \hat{a}(t)\right\rangle}{\left\langle\hat{a}^{\dagger}(t) \hat{a}(t)\right\rangle^{2}}
$$

Let $\tau=0$, (there is no time delay between two arms of the interferometer), the second order correlation can be simplified as:

$$
g^{(2)}(0)=\frac{\left\langle\hat{a}^{\dagger}(t) \hat{a}^{\dagger}(t) \hat{a}(t) \hat{a}(t)\right\rangle}{\left\langle\hat{a}^{\dagger}(t) \hat{a}(t)\right\rangle^{2}}=\frac{\left\langle\left(\hat{a}^{\dagger}\right)^{2} \hat{a}^{2}\right\rangle}{\left\langle\hat{a}^{\dagger} \hat{a}\right\rangle^{2}}
$$

This correlation function is basically the probability of detecting two simultaneous photons, normalized by the probability of detecting two photons at once for a random photon source.

Compare Eq.(T) with the Eq.(A), we can find the relationship between these two equations, which can be written as: 


$$
\frac{V_{n}-\langle n\rangle}{(\langle n\rangle)^{2}}=g^{(2)}(0)-1=\frac{\left\langle\left(\hat{a}^{\dagger}\right)^{2} \hat{a}^{2}\right\rangle-\left\langle\hat{a}^{\dagger} \hat{a}\right\rangle^{2}}{\left\langle\hat{a}^{\dagger} \hat{a}\right\rangle^{2}}
$$

In the classic fields, the second intensity correlation function obeys the following two properties: $g^{(2)}(0) \geq 1$, and $g^{(2)}(0) \leq g^{(2)}(\tau)$

But in the quantum mechanics, some quantum photon sources may violate both these two properties, For example, a source is antibunched and is considered a true single photon source when second photon detection probability reaches zero. For example, a single two-level atom cannot emit two photons at once, and so in this case, $g^{(2)}(0)=0$. This property is easy to be proved because:

$$
g^{(2)}(0)=\frac{\langle n(n-1)\rangle}{\langle n\rangle^{2}}
$$

Where $\mathrm{n}$ represent the observable photon number. For the single photon source, $n=1$, therefore, $g^{(2)}(0)=0$.

\subsection{Single photon source}

The ideal light source for a quantum computing system is one that emits only one photon per given time interval $T=1 / R$, where $R$ is the transmission rate of the source. However, most realistic light sources have a probability of emitting greater than one photon simultaneously, which can be detrimental to quantum key distribution protocols. A single photon source obeys Poissonian statistics, that is it has a reduced probability of emitting a photon if one has already been detected within a certain time interval. [13]

In the classic field, the quality of a single photon source is measured by intensity correlation function $g^{(2)}(\tau)_{\text {classic }}$, which is shown in the equation (3.4.7). While in the 
quantum field, the quality of a single photon source is measured by the probability coherence function $g^{(2)}(\tau)_{\text {quantum, }}$ which is shown in equation (3.4.14). The only restriction on the quantum mechanical correlation function is that it cannot be negative. Also, the correlation function in quantum mechanics does not follow the Poissonian statistics.

As we mentioned in the section (3.4.2), quantum photon sources do not follow the second order correlation properties shown from Eq. (3.4.8) to Eq. (3.4.10), which means there can be a source for which the probability of detecting a second photon is lowest immediately after the first one is detected. Such a quantum photon source is antibunched and can be considered a true single photon source when second photon detection probability decrease to zero. [14]

\subsection{Summary}

In this chapter, we introduce what is the Hong-ou-Mandel effect, and prove this effect by deriving the photon state equation. In order to know how electric field varies at the 50/50 beam splitter, we assumed the plane wave incident in the beam splitter at 45 degree, then observed the properties after the beam splitter by plotting the transmittance and reflection as the function of the beam splitter's width. After doing the preparation work, we introduced the structure of HOM interferometer and how the set up works. To simplify the simulation model for the following chapter, we only consider the source is the single photon source. 


\section{CHAPTER IV}

\section{MANDEL DIP EXPERIMENT AND HOM VISIBILITY}

\subsection{Mandel dip experiment}

Mandel dip experiment is to measure the photon pairs coincidence, based on the HOM interferometer. Before we do the simulation and the experiment of HOM dip, to simplify the simulation model, we need to assume that the incident photons have the same physical properties and the incident angle is 45 degree. As we mentioned in the section (3.3), because of the entanglement of the photon pairs, only one detector in HOM interferometer can detect the photons if the signal and idler photon arrive at the beam splitter at the same time, which means the coincidence of the photon pairs is zero. When we slowly move the beam splitter shown in figure (3.5), the coincidence is no longer zero any more, two photon detectors could count the photons from the single photon source simultaneously. Here is the experimental set up sketch for Mandel dip experiment.

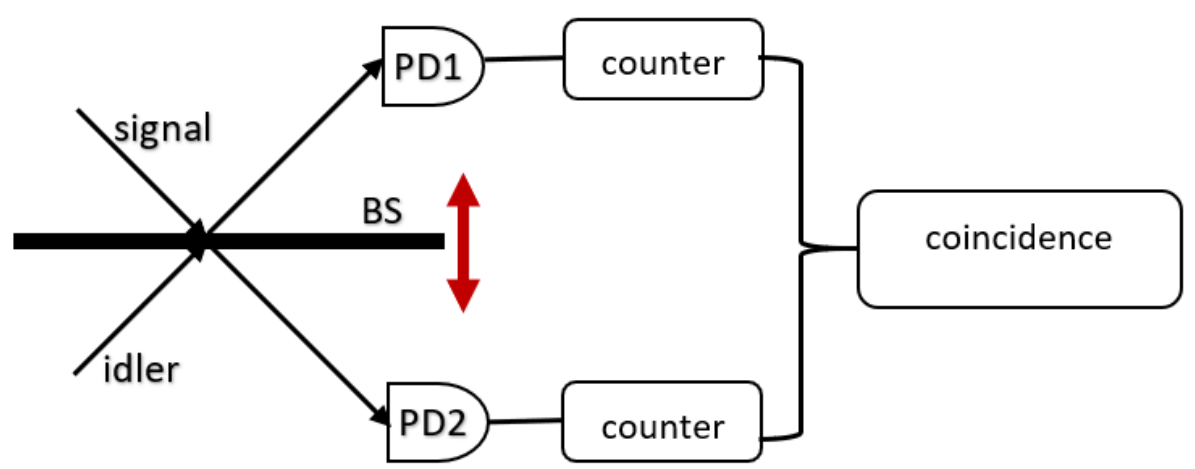

Figure 4.1 set up sketch of Mandel dip experiment

Where PD is the photon detectors, and red arrow represent the shift direction of the beam splitter. Assume both of signal and idler's incident angle are 45 degree. 
In most case, the degeneracy down conversion photons are not monochromatic, then the signal and idler's photon state can be written as:

$$
|\psi\rangle=\int d \omega \phi\left(\omega_{1}, \omega_{0}-\omega_{1}\right)\left|\omega_{1}, \omega_{0}-\omega_{1}\right\rangle
$$

Where $\phi\left(\omega_{1}\right)$ is the wave weight function which has the peak value when $\omega_{1}=\frac{1}{2} \omega_{0}$. The joint probability of the detection of photons at both detectors PD1 and PD2 at times $t$ and $t+\tau$, can be written as: [15] [14]

$$
P_{12}(\tau)=K\left\langle\widehat{E}_{1}^{(-)}(t) \hat{E}_{2}^{(-)}(t+\tau) \hat{E}_{2}^{(+)}(t+\tau) \hat{E}_{2}^{(+)}(t)\right\rangle
$$

Where the $\hat{E}_{1}^{(-)}(t)$ and $\hat{E}_{2}^{(+)}(t)$ means the negative and positive frequency detected by the photon detector, respectively, and $\mathrm{K}$ is some constant for the electric field. Note that the expression of $\hat{E}_{1}^{(+)}(t)$ and $\hat{E}_{2}^{(+)}(t)$ is closely related to the field $\hat{E}_{01}^{(+)}(t)$ and $\hat{E}_{02}^{(+)}(t)$ at the mirror of the interferometer shown in the figure (3.4). If $R=T=50 \%$, then we get:

$$
\begin{aligned}
& \hat{E}_{1}^{(+)}(t)=\sqrt{T} \hat{E}_{01}^{(+)}(t-\tau)+i \sqrt{R} \hat{E}_{02}^{(+)}(t-\tau+\Delta \tau) \\
& \hat{E}_{2}^{(+)}(t)=\sqrt{T} \hat{E}_{02}^{(+)}(t-\tau)+i \sqrt{R} \hat{E}_{01}^{(+)}(t-\tau-\Delta \tau)
\end{aligned}
$$

Where $\tau$ is the propagation time from the mirror to the beam splitter. The different field label for the $\Delta \tau$ argument is because the shift of the beam splitter. If we put equation (4.1.1), (4.1.3) and (4.1.4) to the equation (4.1.2). We can rewrite the equation (4.1.2) as:

$$
P_{12}(\tau)=K|G(0)|^{2}\left[T^{2}|g(\tau)|^{2}+R^{2}|g(2 \Delta \tau-\tau)|^{2}-R T\left(g^{*}(\tau) g(2 \Delta \tau-\tau)\right)+c . c\right](4
$$


Where $G(\tau)$ is the Fourier transform of the wave weight function $\phi\left(\frac{\omega_{0}}{2}+\omega, \frac{\omega_{0}}{2}-\right.$ $\omega) e^{-\omega \tau}$. And we define $g(\tau)=G(\tau) / G(0)$. The equation (4.1.5) shows the possibility both detected by PD1 and PD2 is related to the time interval $\tau$. Both of $G(\tau)$ and $g(\tau)$ are symmetric with $\tau$.

Through some complicated algebra, the expected photon number recorded by coincidence counter can be derived to be: [15]

$$
N_{c}=C\left[R^{2}+T^{2}-2 R T \frac{\int_{-\infty}^{\infty} g(\tau) g(\tau-2 \Delta \tau) d \tau}{\int_{-\infty}^{\infty} g(\tau)^{2} d \tau}\right]
$$

Where $\mathrm{C}$ is a constant, if we consider a specific case that the ratio function $g(\tau)$ is the Gaussian function with the homogeneous broadening, and the bandwidth is $\Delta \omega$. Then, $g(\tau)$ can be written as:

$$
g(\tau)=e^{-\frac{(\Delta \omega \tau)^{2}}{2}}
$$

Take the expression of $g(\tau)$ into the equation (4.1.6), the Eq (4.1.6) yields to:

$$
N_{c}=C\left(R^{2}+T^{2}\right)\left[1-\frac{2 R T}{R^{2}+T^{2}} e^{-\frac{(\Delta \omega \Delta \tau)^{2}}{2}}\right]
$$

Where $\Delta \tau$ is varied with the displacement of the beam splitter. Let's put some realistic values for the parameters of equation (4.1.8) and plot this equation as the function of the BS displacement. Below is a simulation result of the observable photons via a coincidence counter, (Fig. 4.2). 


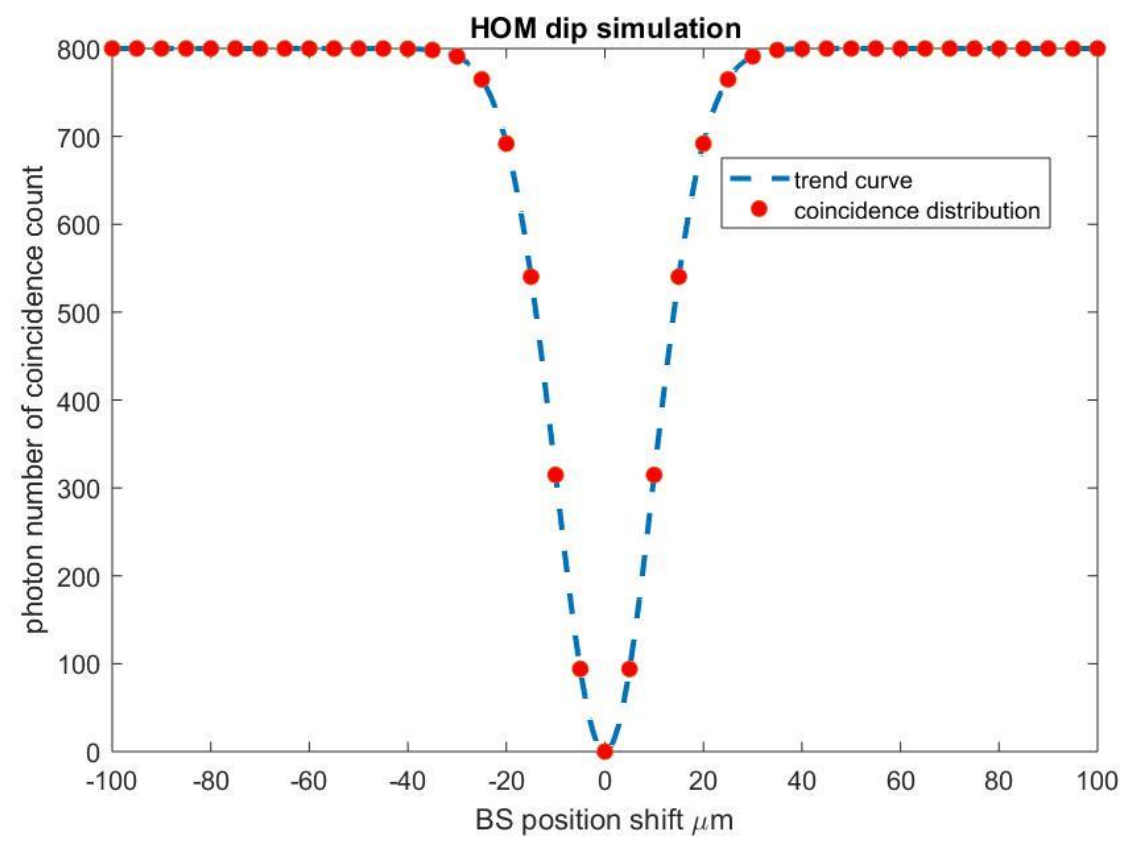

Figure 4.2 simulation of HOM dip, photon coincidence is plotted versus BS position shift

The red spot shows the discrete distribution of the observable coincidence photons, and the dashed line is the trendline curve which we obtain from Eq. (4.1.8) of the observable coincidence photons. Figure (4.3) [15] shows the photons' distribution measured by the experiment, the aberration bar shows the possible aberration of the counting photons. From the figure (4.3) [16], we get that the photons' distribution obey the theoretical expression we get in equation (4.1.8). 


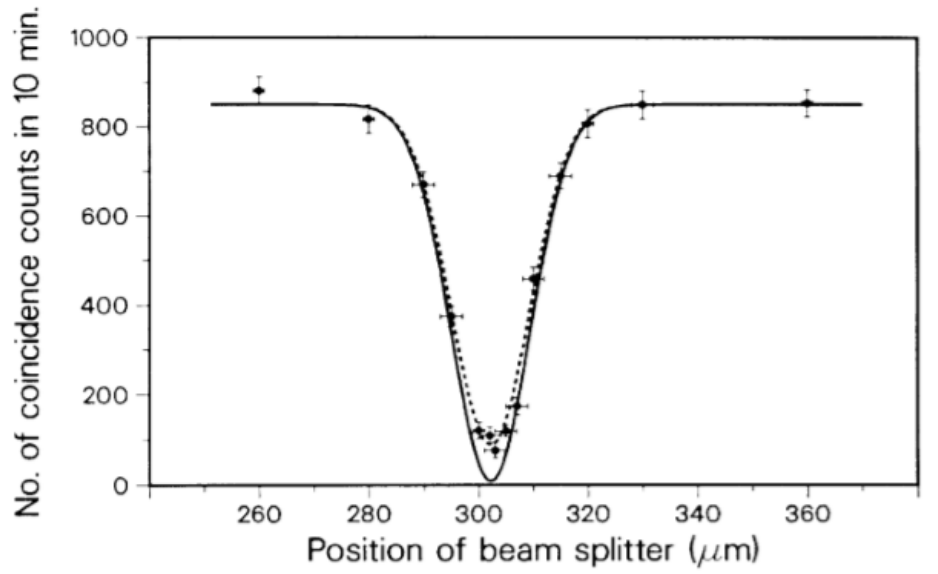

Figure 4.3 HOM dip measured by experiment, aberration bar shows the uncertainty [16]

In this section, we introduce the Mandel dip experiment set up, and derive the observable coincidence photons expression from the photon state distribution. Also, we do the simulation of coincidence distribution and compare this result to the real experimental result. From comparing the simulation figure to the experiment figure, we find they match well.

\subsection{Characterizing entanglement in photon pairs}

A single photon pairs' state from SPDC process can be expressed as a weighted sum of creation operators acting on vacuum as follows:

$$
\begin{aligned}
|\psi\rangle=\sum_{\mu_{s}} \sum_{\mu_{i}} & \int_{0}^{\infty} d \omega_{s} \int_{0}^{\infty} d \omega_{i} \int d k_{s}^{\perp} \int d k_{i}^{\perp} S\left(\omega_{s}, k_{s}^{\perp}, \mu_{s} ; \omega_{i}, k_{i}^{\perp}, \mu_{i}\right) \\
& \left.\times \hat{a}_{k_{s}^{\perp} \mu_{s}}^{\dagger}\left(\omega_{s}\right) \hat{a}_{k_{i}^{\perp} \mu_{i}}^{\dagger}\left(\omega_{i}\right) \mid \text { vacuum }\right\rangle
\end{aligned}
$$

Where the function $S\left(\omega_{s}, k_{s}^{\perp}, \mu_{s} ; \omega_{i}, k_{i}^{\perp}, \mu_{i}\right)$ not only represent the photon pairs amplitude, but also contains all the freedom of the photon pairs: frequency, transverse momentum and polarization. The single photon pairs' probability amplitude depends on 
the form of the electromagnetic field used as a pump in the down-conversion process, and on the so-called phase matching function, defined by the optical properties of the crystal. A useful tool for analyzing the entanglement present in the continuous degree of freedom is to carry out a Schmidt decomposition of the spatial-temporal-polarization state amplitude $S\left(\omega_{s}, k_{s}^{\perp}, \mu_{s} ; \omega_{i}, k_{i}^{\perp}, \mu_{i}\right)$ into a sum of products of functions. This function is also called as Schmidt decomposition function, which gives:

$$
S\left(\omega_{s}, k_{s}^{\perp}, \mu_{s} ; \omega_{i}, k_{i}^{\perp}, \mu_{i}\right)=\sum_{n} \sqrt{\lambda_{n}} \psi_{n}\left(\omega_{s}, k_{s}^{\perp}, \mu_{s}\right) \phi_{n}\left(\omega_{i}, k_{i}^{\perp}, \mu_{i}\right)
$$

Where $\omega_{s}, k_{s}^{\perp}, \mu_{s}$ represent the wavelength, transverse wavevector and polarization of the photon pairs. In general, obtaining such a Schmidt decomposition entails solving certain integral equations. By approximating the "Sinc" function in the phase matching function as a Gaussian it becomes possible to carry out the decomposition analytically in certain cases. It is found that the resulting entanglement does not necessarily involve the infinitely many degrees of freedom in the frequency-space-polarization continuum with typically only a finite number of Schmidt modes having appreciable eigenvalue magnitudes. The amount of entanglement can be conveniently quantified by the cooperativity parameter [5] defined in terms of the Schmidt eigenvalues as: [17]

$$
K=\frac{1}{\sum_{k} \lambda_{k}^{2}}
$$

The eigenvalue $K$ gives an indication of the number of active Schmidt mode pairs, which in turn is a measure of how much entanglement is present in the photon pairs. A twophoton state for which the cooperativity assumes its minimum allowed value $K=1$ 
represents a state in which there is a single pair of Schmidt modes and therefore exhibits no spectral entanglement.

Figure (4.4) shows the relationship between signal frequency and idler frequency under the type I and type II SPDC, respectively [17].
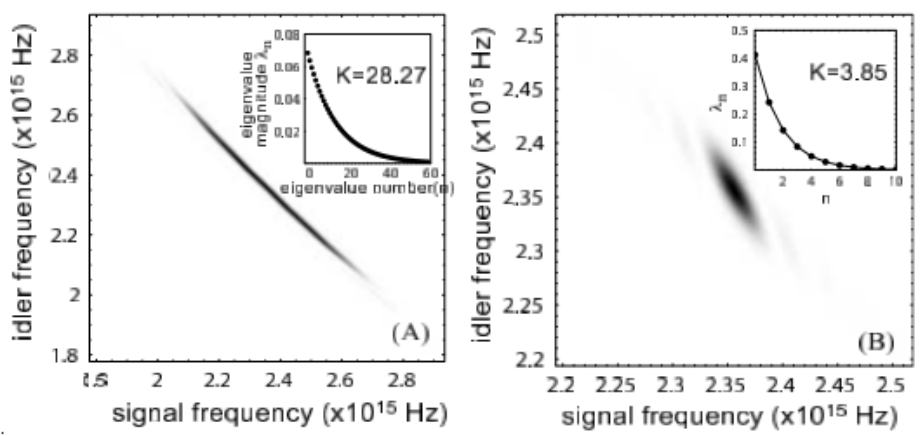

Figure 4.4 plot idler frequency as the function of signal frequency under the different $K$ value [17]

In Figure (4.4), it is seen that type I PDC has a higher degree of spectral entanglement as quantified by the value of the cooperativity parameter K.

For typical experimental parameters both type I and type II SPDC (mentioned in chapter III), though particularly the former, exhibit large spectral entanglement, as quantified by the large value of $\mathrm{K}$. But does that mean the higher value of $\mathrm{K}$ is the only desirable requirement? The answer to this question depends on what experiment we wish to perform. In most cases, higher value of K represents the tight photon's correlation, but in some special cases, we may need the anticorrelation of the photon pairs. 


\subsection{HOM visibility}

\subsubsection{Introduction of HOM visibility}

From the introduction in section (3.3) and the simulation in section (4.2), single photon pairs are created using the process of parametric down conversion (PDC) and were then sent through two arms of the HOM interferometer. From the observable coincidence figure (4.2), the frequency of photon pairs cannot distinguish at all when the coincidence counter decrease to zero. That means the single photon pairs is entangled with the same frequency and the visibility is zero. However, the photon pairs will not entangle and can be perfectly distinguish when the beam splitter shift far away. The questions now become how we can define the photon visibility when only some of the outcome photons can be distinguished but the other photons cannot be distinguished?

The phenomenon of nonzero probability of finding a particle propagating through a potential barrier is a unique consequence of quantum mechanics that has been widely researched in modern science.

The degree of single photon interference can be measured by the visibility of the HOM dip, which can be roughly defined by: [18]

$$
V=\frac{C_{\max }-C_{\min }}{C_{\max }+C_{\min }}
$$

where $C_{\max }$ is the maximum coincidence that can be observed and $C_{\min }$ is the minimum coincidences that can be observed by the detectors. It is important to note that in practice the visibility is never exactly equal to unity. Any mismatches in the spectral function and misalignments of the setup decrease interference effects. Since most beam splitters suffer losses and do not split the light perfectly, it is yet another reason for this imbalance. 
Our goal is to develop photon sources that produce photons in well specified single spatial-temporal modes thus eliminating the usual need for filtering: the resulting engineered photons would have identical mode structures, with in principle no distinguishing information residing in any degree of freedom, thus ensuring high visibility interference.

\subsubsection{Loss of HOM visibility}

Here I begin with modeling of the PDC process that creates a pair of identical photons born simultaneously. The state of the PDC generated single photon pairs is given by:

$$
\begin{gathered}
|\psi(t)\rangle=K_{1}|1,0\rangle|1,0\rangle+ \\
K_{2} \sum_{\omega 1} \sum_{\omega 2} \varphi\left(\omega_{1}, \omega_{2}\right) \frac{\sin \frac{1}{2}\left(\omega_{1}+\omega_{2}-\omega_{0}\right) t}{\frac{1}{2}\left(\omega_{1}+\omega_{2}-\omega_{0}\right)} \exp \left(i\left(\omega_{1}+\omega_{2}-\omega_{0}\right) t / 2\right)\left|\omega_{s}\right\rangle\left|\omega_{i}\right\rangle
\end{gathered}
$$

Where $\varphi\left(\omega_{1}, \omega_{2}\right)$ is the spectral distribution function of the two-photon wavepacket, and $\omega_{1}, \omega_{2}$

are respective photon frequencies. In the case of unmodulated twin photons, $\varphi\left(\omega_{1}, \omega_{2}\right)$ is

symmetric and has a maximum at $\frac{\omega_{0}}{2}$. [11]

As what mentioned in the section 4.1, the expected photon number recorded by coincidence counter can be derived to be: [15]

$$
N_{c}=C\left[R^{2}+T^{2}-2 R T \frac{\int_{-\infty}^{\infty} g(\tau) g(\tau-2 \Delta \tau) d \tau}{\int_{-\infty}^{\infty} g(\tau)^{2} d \tau}\right]
$$


Where $\mathrm{C}$ is a constant, if we consider a specific case that the ratio function $g(\tau)$ is the Gaussian function (shown in 4.1.7) with the homogeneous broadening, and the bandwidth is $\Delta \omega$. Then, the coincidence function (4.3.3) yields to:

$$
N_{c}=C\left[1-\frac{2 R T}{R^{2}+T^{2}} e^{-\frac{(\Delta \omega \Delta \tau)^{2}}{2}}\right]
$$

Equation (4.3.4) has already proved in the previous part. Now, let's consider the condition when the loss exist in the nonlinear crystal, which means the reflectivity $\mathrm{R}$ and transmissivity $\mathrm{T}$ are not equal to $50 \%$ and the same time $(R+T<1)$.

From equation (4.3.4), if there is no shift of the beam splitter $(\Delta \tau=0)$, the coincidence function becomes zero when $R=T=50 \%$. However, if $R+T<1$, the amplitude factor $2 R T /\left(R^{2}+T^{2}\right)$ is not equal to 1 , then whole exponential term $\frac{2 R T}{R^{2}+T^{2}} e^{-\frac{(\Delta \omega \Delta \tau)^{2}}{2}} \neq 1$ even if $\Delta \tau=0$. Because the exist of the loss, the coincidence is not zero when there is no shift of the beam splitter, corresponding to the reduction of the visibility.

Direct measurements of the beam-splitter reflectivity and transmissivity show that $R+$ $T=0.9,(R=0.5, T=0.4$. $)$ which makes the combination $2 R T /\left(R^{2}+T^{2}\right)=0.975$, and implies that $N_{c}$, should fall close to zero when $\Delta \tau=0$. That it does not fall quite that far is probably due to a slight lack of overlap of the signal and idler fields admitted by the two pinholes, causing less than perfect destructive interference. The solid curve in Figure (4.5) is based on Eq. (4.3.4) with $\Delta \omega \sim 10^{13} \mathrm{~Hz}$. And we identify $c \Delta \tau$ with the beamsplitter displacement in micrometers. For the dashed curve the factor $2 R T /\left(R^{2}+T^{2}\right)$ was multiplied by 0.9 to allow for less than perfect overlap of the signal and idler 
photons. It will be seen that, except for the minimum, Eq. (4.3.4) is obeyed quite well, corresponding to a coherence time of about $100 \mathrm{fs}$.

Figure (4.5) shows the example how the reflectivity and transmissivity loss affects the visibility and coincidence function.

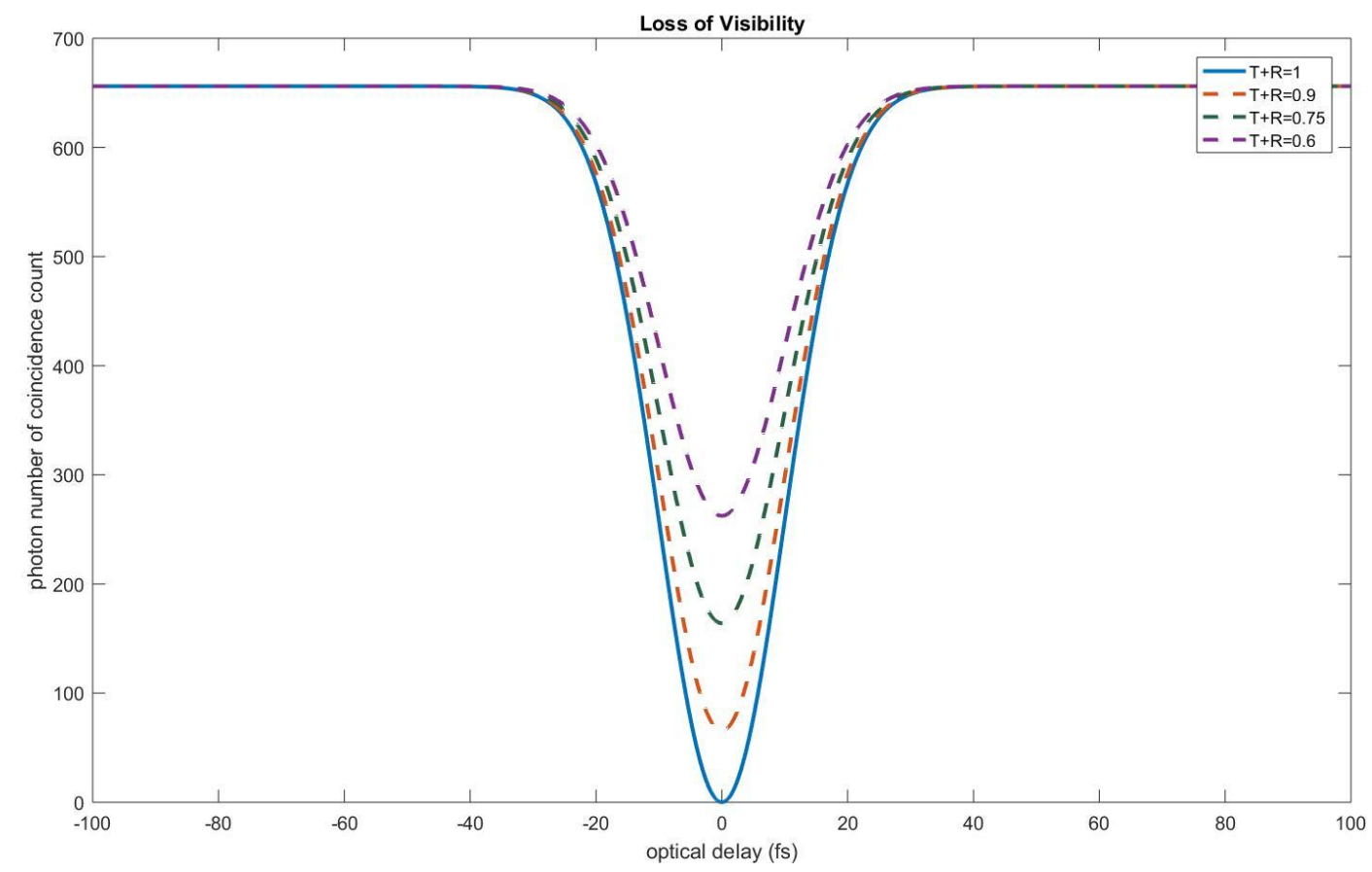

Figure 4.5. Loss of visibility. Plotted different value of $T+R$

From the figure (4.5) we find with increasing the loss of the reflectivity and transmittivity, the coincidence that both of detectors can detect the photons is increasing, thus, the visibility is reducing. This phenomenon shows the loss in the nonlinear crystal will cause the dramatic reduction of photon visibility.

\subsubsection{Asymmetric dispersion of wave packet}

From the analysis of section 4.3.2, we know the because of the absorption of nonlinear crystal $(T+R<1)$, resulting in the reduction of the HOM visibility. Besides absorption of the nonlinear crystal, there is another reason causing the loss of the visibility: unideal 
phase-matched photons. Consider the photon pairs are not phase matched after SPDC process, their frequency is not symmetric with the central frequency.

As shown in Eq. (4.3.2), $\varphi\left(\omega_{1}, \omega_{2}\right)$ is the spectral distribution function of the two-photon wavepacket, and $\omega_{1}, \omega_{2}$ are respective photon frequencies. In this case of because of the misphase-matched of the photon pairs, $\varphi\left(\omega_{1}, \omega_{2}\right)$ is not symmetric at $\frac{\omega_{0}}{2}$. Thus, the Fourier transform of the weight function $\varphi\left(\frac{\omega_{0}}{2}+\omega_{1}, \frac{\omega_{0}}{2}-\omega_{2}\right)$ becomes:

$$
G(\tau)=\int \varphi\left(\frac{\omega_{0}}{2}+\omega_{1}, \frac{\omega_{0}}{2}-\omega_{2}\right) e^{-i \omega t} d \omega
$$

Where $g(\tau)$ is the Gaussian function with the bandwidth $\Delta \omega=\omega_{1}-\omega_{2}$, and $g(\tau)=$ $G(\tau) / G(0)$. In this case, $g(\tau)$ is no longer reach the maximum value when $\tau=0$. Here is the sketch of the asymmetric wave-packet.

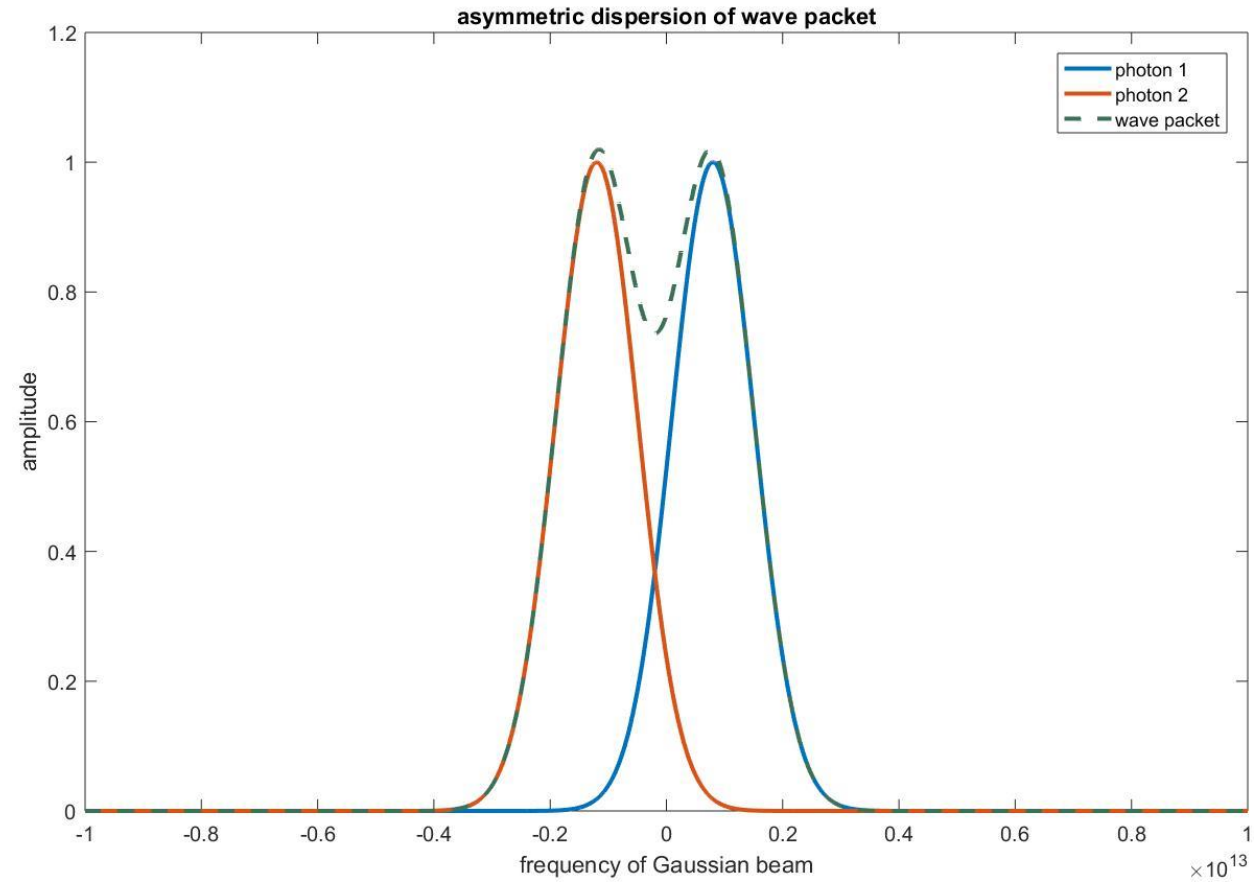

Figure 4.6 Sketch of asymmetric wave dispersion 
Because of the asymmetric dispersion, the wave-packet Gaussian function written in equation (4.1.7) yields to

$$
g(\tau)=e^{-\frac{\left[\left(\omega_{1}-\omega_{0}\right)^{2}+\left(\omega_{2}-\omega_{0}\right)^{2}\right] \tau^{2}}{2}}
$$

Take equation (4.3.6) into equation (4.3.6), then, the coincidence function can be written as:

$$
N_{c}=C\left[1-\frac{2 R T}{R^{2}+T^{2}} e^{-\frac{\left[\left(\omega_{1}-\omega_{0}\right)^{2}+\left(\omega_{2}-\omega_{0}\right)^{2}\right] \tau^{2}}{2}}\right]
$$

Figure (4.7) shows the Mandel dip function for the asymmetric photon wave. The dash curve is the symmetric condition while the solid curve shows the asymmetric condition.

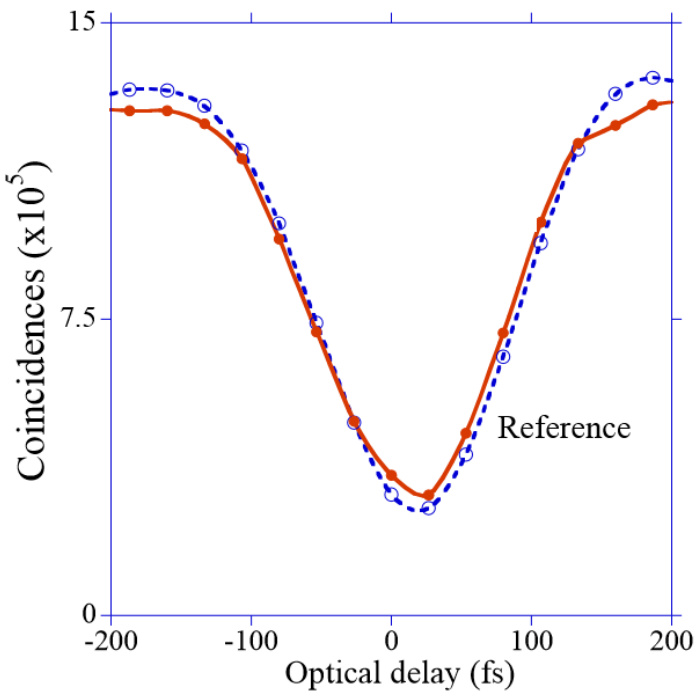

Figure 4.7 HOM dip shift and visibility loss

From figure (4.7), we find the coincidence is still not zero when $\Delta \tau=0$. That shows the loss of the visibility exists in when two photons are not perfectly phase-matched. Also, from figure (4.7), the curve is not centered at the " 0 " point because of the asymmetric dispersion of two photon wave. 


\subsection{Summary}

In the chapter IV, we do the Mandel dip experiment on the base of HOM interferometer, and we explain the generation of the HOM dip is due to the displacement of the beam splitter in the interferometer. Meanwhile, we derive the distribution of the observable photons coincidence from the expression of photon state, and simulate this result by Matlab. On the base of HOM dip experiment result, we give the definition of the photon visibilities. The observed visibility difference of an HOM dip can be used to describe a change in a spectral function of a signal compared to idler. In order to explore what factors will influence the photon visibility, we give the criteria for photon pairs' entanglement, then obtain the eigenvalue K of the Schmidt equation to measure how much entanglement is present in the photon pairs. At last, plotting the idler frequency as the function of the signal frequency under the different $K$ value to show how the eigen value affect the correlation. In the last section of this chapter, we describe another dimension of HOM measurements that utilizes the change in the visibility of the HOM profile.

In section 4.3, we introduce the definition of HOM visibility and analyze two main reasons that would cause the loss of the visibility. In order to explain the loss of the visibility in details, we plot the coincidence function to show the difference between the Mandel dip without loss and with loss. Because the loss exists in photon interference, the dip cannot reach to zero even if beam-splitter does not shift, corresponding to the reduction of the visibility. 


\section{CHAPTER V \\ CONCLUSION AND FUTURE WORK}

\subsection{Conclusion}

The HOM interferometer has proven to be a valuable research tool in the field of quantum optics. One of the great properties of this interferometer is a quantitative measurement of distinguishability of single photons. In addition, HOM interferometer can be used as a super sensitive sensor to measure various classical properties though the quantum effect.

In my thesis research I aim at understanding how HOM interferometer works and the reason why the HOM dip generate. In Chapter II I describe several nonlinear parametric conversions and the phase matching process as the background knowledge of the SPDC. By plotting the phase matching efficiency as the function of the pump wavelength, we can calculate the bandwidth of the phase matching. In Chapter III, I introduce some basic concept of the quantum mechanics, for example, what is the HOM effect, how can we define the order of the photons' correlation, and what is the photon antibunching phenomenon. Also, we explain how HOM interferometer works, and simplify introduce the coincidence of the observable photons. In chapter IV, we derive the distribution of the observable photons' coincidence from the expression of photon state. Also, we give the definition of the photon distinguishability by measuring the changes in visibility of an HOM dip. In order to explore what factors will influence the photon visibility, we give the criteria for photon pairs' entanglement, then obtain the eigenvalue K of the Schmidt equation to measure how much entanglement is present in the photon pairs. 


\subsection{Future work}

In this project, once we do some quantum calculation and derivation, we only consider about the single photon source, which is the simplest model for the photon's correlation. In the future work, we consider try to do some calculation about what if the photons are not totally identical. Also, we only consider the Type I $(e \rightarrow o+o)$ SPDC process when focusing the actual example of two photons' state. Is the visibility of the photon distribution under Type II $(e \rightarrow o+e)$ SPDC process different from the Type I? That's also a good question to be considered in the future work. And in my work, I only consider a single SPDC process of photon pairs, how about the pump photons go through several SPDC process? In the future research, I need to build the criteria for the design of photon states to be used in multiple-crystal interferometry. 


\section{REFERENCES}

[1] Q. Algorithms, "https://quantumalgorithms.herokuapp.com/299/paper/node21.html," [Online].

[2] P. Shor, "Algorithms for quantum compution: discrete logarithms and factoring," in Proceedings 35th Annual Symposium on Foundations of Computer Science.

[3] Peter E. Powers. Joseph W. Haus, in Fundamentals of nonlinaer optics, 2018, pp. 124-140.

[4] Peter E. Power. Joseph. W. Haus, in Fundemental of nonlinear optics, 2018, pp. 6768.

[5] J. W. H. \&. P. Power, "fundamental of nonlinear optics," 2017 second edition, pp. 124-126.

[6] https://www.rp-photonics.com/effective_nonlinear_coefficient.html, "RP Photonics Encyclopedia," RP Photonics . [Online].

[7] Peter E. Power. Joseph W. Haus, in fundemental of nonlinear optics, 2018, p. 444.

[8] X. P. Hu, P. Xu and S. Zhu, "Engineered quasi-phase matching for laser techniques," in Photonic Research, 2013.

[9] P. E. P. J. W. Haus, in Fundamental of nonlinear optics , pp. 164-167.

[10] R. Boyd, in Nonlinear Optics (Third edition), New York, academic press, 2008 , pp. 79-88.

[11] N. Borjemscaia, "Hong Ou Mandel Interferometer: A Quantum Measurement Tool," 2012. 
[12] P.Chen, C.Shu, X.Guo and M. T. Loy, "Measuring the biphoton temporal wave function with polarization-dependent and time-resolved two photon interference," Physical review, p. 114, 2015.

[13] R. Loudon, "The Quantum Theory of Light," Clarendon Press, 1983.

[14] H. J. Kimble, M. Dagenais, and L. Mandel, " Photon antibunching in resonance," Phys. Rev. Lett, pp. 691-695, 1977.

[15] C. K. Hong, Z. Y. Ou, and L. Mandel, "Measurement of Subpicosecond Time Intervals between Two Photons by Interference," physical review letter, pp. 20442046, 1987.

[16] K. B. A. I. W. A.B. U’Rena, " Photon Engineering for Quantum Information Processing," quantum physics, 3052003.

[17] A.B. U'Rena, K. Banaszek and I.A. Walmsley, "Photon Engineering for Quantum Information Processing," Clarendon Laboratory, Oxford University, Oxford, OXI 3PU, UK, May 2003.

[18] A. M. Bran'czyk, "Hong-Ou-Mandel Interference," Perimeter Institute for Theoretical Physics, , 2017.

[19] E. W. L. Mandel, in Optical coherence and quantum optics, Cambridge University Press. 


\section{APPENDIX}

Matlab Code used in this work

\section{Chapter II, find the phase matching angle}

clc;

clear all;

close all;

lambda1 $=0.532$;

lambda2=0.6;

lambda3 $=(1 / \text { lambda1 }-1 / \text { lambda2 })^{\wedge}(-1)$;

no2 $=\operatorname{sqrt}\left(4.9048+0.11775 /\left(\operatorname{lambda} 2^{\wedge} 2-0.218^{\wedge} 2\right)-0.027153 *\right.$ lambda $\left.2^{\wedge} 2\right)$;

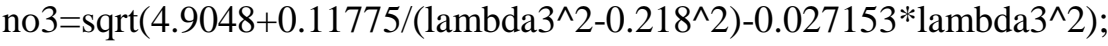

no1 $=\operatorname{sqrt}\left(4.9048+0.11775 /\left(\right.\right.$ lambda $\left.1^{\wedge} 2-0.218^{\wedge} 2\right)-0.027153 *$ lambda $\left.1^{\wedge} 2\right)$;

$\mathrm{nz} 1=\operatorname{sqrt}\left(4.582+0.09921 /\left(\mathrm{lambda} 1^{\wedge} 2-0.211^{\wedge} 2\right)-0.02194 * 1 \mathrm{ambda} 1^{\wedge} 2\right)$;

theta $=0: 0.001: \mathrm{pi} / 2$;

ne $1=\operatorname{sqrt}\left(\left((\cos (\right.\right.$ theta $)) .^{\wedge} 2 / n o 1^{\wedge} 2+(\sin ($ theta $\left.\left.)) .^{\wedge} 2 / n z 1^{\wedge} 2\right) .^{\wedge}(-1)\right)$;

deltak=2*pi*(ne1./lambda1-no2/lambda2-no3/lambda3);

$\mathrm{y}=0 *$ theta;

plot(theta/pi*180,deltak);

hold on

plot(theta/pi*180,y);

title('Type1 pahse matching, Idelta $\mathrm{k}$ versus angle \theta');

ylabel('ldeltak \mum^^-1');

xlabel('angleltheta degrees');

legend('e-o+o','\deltak=0');

\section{Chapter II, phase matching efficiency of SPDC}

\section{(1) Collinear condition}

clc;

clear all;

close all;

$\mathrm{T} 0=24.5 ;$ 
$\mathrm{T}=45$

$\mathrm{F}=(\mathrm{T}-\mathrm{T} 0) *(\mathrm{~T}+\mathrm{T} 0+546) ;$

lambda_p=0.8;

lambda_s=1.6;

lambda_I=(1/lambda_p-1/lambda_s $)^{\wedge}(-1)$;

$\%$ only consider the polarization in $\mathrm{z}$ direction

$\mathrm{nz} \_\mathrm{p}=\operatorname{sqrt}\left(5.35583+4.629 \mathrm{e}-7 * \mathrm{~F}+\left((0.100473+3.862 \mathrm{e}-8 * \mathrm{~F}) /\left(\right.\right.\right.$ lambda_p $\mathrm{p}^{\wedge} 2-$

$\left.\left.0.20692^{\wedge} 2\right)\right)+\left(100+2.657 \mathrm{e}-5^{*} \mathrm{~F}\right) /\left(\right.$ lambda_p $\left.\left.{ }^{\wedge} 2-128.806\right)-0.015334 * 1 a m b d a \_\mathrm{p}^{\wedge} 2\right)$;

nz_s $=\operatorname{sqrt}\left(5.35583+4.629 \mathrm{e}-7 * \mathrm{~F}+\left((0.100473+3.862 \mathrm{e}-8 * \mathrm{~F}) /\left(\right.\right.\right.$ lambda_s $\mathrm{s}^{\wedge} 2-$

$\left.\left.0.20692^{\wedge} 2\right)\right)+\left(100+2.657 \mathrm{e}-5^{*} \mathrm{~F}\right) /\left(\right.$ lambda_s $\left.\left.\mathrm{s}^{\wedge} 2-128.806\right)-0.015334 * \mathrm{lambda} \_\mathrm{s}^{\wedge} 2\right)$;

nz_I $=$ sqrt $(5.35583+4.629 \mathrm{e}-7 * \mathrm{~F}+((0.100473+3.862 \mathrm{e}-8 * \mathrm{~F}) /($ lambda_I^ $2-$

$\left.0.20692^{\wedge} 2\right)+\left(100+2.657 \mathrm{e}-5^{*} \mathrm{~F}\right) /($ lambda_I^2 $2-128.806)-0.015334 *$ lambda_I^2);

lambdaC=1/(nz_p/lambda_p-nz_s/lambda_s-nz_I/lambda_I);\%calculate capital lambda when k=0

\%see what will happen about capital lambda

lambda_p1=0.5:0.001:2.5;

lambda_s1=2*lambda_p1;\%assume signal and idler are degeneracy

lambda_I1=2*lambda_p1;

nz_p1 $=$ sqrt $\left(5.35583+4.629 \mathrm{e}-7 * \mathrm{~F}+\left((0.100473+3.862 \mathrm{e}-8 * \mathrm{~F}) . /\left(1 \mathrm{ambda} \_\mathrm{p} 1 .^{\wedge} 2-\right.\right.\right.$

$\left.\left.0.20692^{\wedge} 2\right)\right)+\left(100+2.657 \mathrm{e}-5^{*} \mathrm{~F}\right) . /($ lambda_p1.^2-128.806)-0.015334*lambda_p1.^2);

nz_s1 $=$ sqrt $\left(5.35583+4.629 \mathrm{e}-7 * \mathrm{~F}+\left((0.100473+3.862 \mathrm{e}-8 * \mathrm{~F}) . /\left(1 \mathrm{ambda} \_\mathrm{s} 1 .^{\wedge} 2-\right.\right.\right.$

$\left.\left.0.20692^{\wedge} 2\right)\right)+\left(100+2.657 \mathrm{e}-5^{*} \mathrm{~F}\right) . /($ lambda_s1.^2-128.806)-0.015334*lambda_s1.^2);

nz_I1 $=\operatorname{sqrt}\left(5.35583+4.629 \mathrm{e}-7 * \mathrm{~F}+\left((0.100473+3.862 \mathrm{e}-8 * \mathrm{~F}) . /\left(\right.\right.\right.$ lambda_I1 $.^{\wedge} 2-$

$\left.\left.0.20692^{\wedge} 2\right)\right)+(100+2.657 \mathrm{e}-5 * \mathrm{~F}) . /($ lambda_I1.^2-128.806)-0.015334*lambda_I1.^2);

lambdaC1=1./(nz_p1./lambda_p1-nz_s1./lambda_s1-nz_I1./lambda_I1);

figure(1)

plot(lambda_p1,lambdaC1)

title('relationship between Vlambdap and capitalllambda');

xlabel('pump wavelength um');

ylabel('capital \lambda um');

$\mathrm{L}=20000$;

lambdap=1.1:0.0001:1.6;\%regard lambdap as a variable

lambdas=2*lambdap;\%assume signal and idler are degeneracy 
lambdaI=2*lambdap;

nzp $=\operatorname{sqrt}\left(5.35583+4.629 \mathrm{e}-7 * \mathrm{~F}+\left((0.100473+3.862 \mathrm{e}-8 * \mathrm{~F}) . /\left(\right.\right.\right.$ lambdap. $.^{\wedge}-$

$\left.\left.0.20692^{\wedge} 2\right)\right)+\left(100+2.657 \mathrm{e}-5^{*} \mathrm{~F}\right) . /\left(\right.$ lambdap. $\left.{ }^{\wedge} 2-128.806\right)-0.015334 *$ lambdap. $\left.{ }^{\wedge} 2\right)$;

nzs $=\operatorname{sqrt}\left(5.35583+4.629 \mathrm{e}-7 * \mathrm{~F}+\left(\left(0.100473+3.862 \mathrm{e}-8^{*} \mathrm{~F}\right) . /\left(\right.\right.\right.$ lambdas. $\left.\left.{ }^{\wedge} 2-0.20692^{\wedge} 2\right)\right)+(100+2.657 \mathrm{e}-$ $\left.5^{*} \mathrm{~F}\right) . /($ lambdas.^2-128.806)-0.015334*lambdas.^2);

nzI $=$ sqrt $\left(5.35583+4.629 \mathrm{e}-7 * \mathrm{~F}+\left((0.100473+3.862 \mathrm{e}-8 * \mathrm{~F}) . /\left(\right.\right.\right.$ lambdaI. $\left.\left.{ }^{\wedge} 2-0.20692^{\wedge} 2\right)\right)+(100+2.657 \mathrm{e}-$ $5 * \mathrm{~F}) . /($ lambdaI.^2-128.806)-0.015334*lambdaI.^2);

deltak=2*pi*(nzp./lambdap-nzs./lambdas-nzI./lambdaI)-2*pi/35.388;\%condition of phase matching

efficiency $=(\operatorname{sinc}(\operatorname{deltak} * L / 2)) .^{\wedge} 2$;

figure(2)

plot(lambdap,efficiency)

title(' $2 \mathrm{~cm}$ lithium niobate bandwidth');

xlabel('pump wavelength lambda(\mum)');

ylabel('leta quasi phase matching efficiency');

\section{(2) Noncollinear condition}

clc;

clear all;

close all;

$\mathrm{T} 0=24.5$;

$\mathrm{T}=45$;

$\mathrm{F}=(\mathrm{T}-\mathrm{T} 0) *(\mathrm{~T}+\mathrm{T} 0+546)$;

theta $=3 * \mathrm{pi} / 180$;

lambda_p=0.8;

lambda_s=1.6;

lambda_I=(1/lambda_p-1/lambda_s $)^{\wedge}(-1)$;

\%only consider the polarization in $\mathrm{z}$ direction

$\mathrm{nz} \_\mathrm{p}=\operatorname{sqrt}\left(5.35583+4.629 \mathrm{e}-7 * \mathrm{~F}+\left((0.100473+3.862 \mathrm{e}-8 * \mathrm{~F}) /\left(\right.\right.\right.$ lambda $\_\mathrm{p}^{\wedge} 2-$

$\left.\left.0.20692^{\wedge} 2\right)\right)+(100+2.657 \mathrm{e}-5 * \mathrm{~F}) /\left(\right.$ lambda_p$\left.{ }^{\wedge} 2-128.806\right)-0.015334 *$ lambda_p $\left.\mathrm{p}^{\wedge} 2\right)$;

$\mathrm{nz} \_\mathrm{s}=\operatorname{sqrt}\left(5.35583+4.629 \mathrm{e}-7 * \mathrm{~F}+\left((0.100473+3.862 \mathrm{e}-8 * \mathrm{~F}) /\left(1 \mathrm{ambda} \_\mathrm{s}{ }^{\wedge} 2-\right.\right.\right.$

$\left.\left.0.20692^{\wedge} 2\right)\right)+(100+2.657 \mathrm{e}-5 * \mathrm{~F}) /\left(\right.$ lambda_s $\left.{ }^{\wedge} 2-128.806\right)-0.015334 *$ lambda_s $\left.{ }^{\wedge} 2\right)$;

nz_I $=$ sqrt $(5.35583+4.629 \mathrm{e}-7 * \mathrm{~F}+((0.100473+3.862 \mathrm{e}-8 * \mathrm{~F}) /($ lambda_I^2-

$\left.\left.0.20692^{\wedge} 2\right)\right)+\left(100+2.657 \mathrm{e}-5^{*} \mathrm{~F}\right) /\left(\right.$ lambda_I$\left.{ }^{\wedge} 2-128.806\right)-0.015334 *{ }^{2}$ lambda_I$\left.{ }^{\wedge} 2\right)$; 
lambda=1/(nz_p/lambda_p-(nz_s/lambda_s+nz_I/lambda_I) $* \cos ($ theta $)) ; \%$ calculate capital lambda when $\mathrm{k}=0$

\%see what will happen about capital lambda

lambda_p1=0.5:0.0001:3;

lambda_s1=2*lambda_p1;\%assume signal and idler are degeneracy

lambda_I1=2*lambda_p1;

nz_p1=sqrt $(5.35583+4.629 \mathrm{e}-7 * \mathrm{~F}+((0.100473+3.862 \mathrm{e}-8 * \mathrm{~F}) . /($ lambda_p1.^2-

$\left.\left.0.20692^{\wedge} 2\right)\right)+(100+2.657 \mathrm{e}-5 * \mathrm{~F}) . /($ lambda_p1.^2-128.806)-0.015334*lambda_p1.^2);

nz_s1 $=\operatorname{sqrt}(5.35583+4.629 \mathrm{e}-7 * \mathrm{~F}+((0.100473+3.862 \mathrm{e}-8 * \mathrm{~F}) . /($ lambda_s1.^2-

$\left.\left.0.20692^{\wedge} 2\right)\right)+\left(100+2.657 \mathrm{e}-5^{*} \mathrm{~F}\right) . /($ lambda_s1.^2-128.806)-0.015334*lambda_s1.^2);

$\mathrm{nz} \_\mathrm{I} 1=\operatorname{sqrt}(5.35583+4.629 \mathrm{e}-7 * \mathrm{~F}+((0.100473+3.862 \mathrm{e}-8 * \mathrm{~F}) . /($ lambda_I1.^2-

$\left.\left.0.20692^{\wedge} 2\right)\right)+(100+2.657 \mathrm{e}-5 * \mathrm{~F}) . /($ lambda_I1.^2-128.806)-0.015334*lambda_I1.^2);

lambdaC=1./(nz_p1./lambda_p1-(nz_s1./lambda_s1+nz_I1./lambda_I1)*cos(theta));

figure(1);

plot(lambda_p1,lambdaC)

title('relationship between Vlambdap and capital \lambda');

xlabel('pump wavelength');

ylabel('capital \lambda');

$\mathrm{L}=20000$;

lambdap=1.2:0.0001:1.6;\%regard lambdap as a variable

lambdas $=2 *$ lambdap;\%assume signal and idler are degeneracy

lambdaI=2*lambdap;

nzp $=\operatorname{sqrt}\left(5.35583+4.629 \mathrm{e}-7 * \mathrm{~F}+\left((0.100473+3.862 \mathrm{e}-8 * \mathrm{~F}) . /\left(\right.\right.\right.$ lambdap. ${ }^{\wedge} 2-$

$\left.\left.0.20692^{\wedge} 2\right)\right)+\left(100+2.657 \mathrm{e}-5^{*} \mathrm{~F}\right) . /\left(\right.$ lambdap. $\left.{ }^{\wedge} 2-128.806\right)-0.015334 *$ lambdap. $\left.{ }^{\wedge} 2\right)$;

nzs $=\operatorname{sqrt}\left(5.35583+4.629 \mathrm{e}-7 * \mathrm{~F}+\left(\left(0.100473+3.862 \mathrm{e}-8^{*} \mathrm{~F}\right) . /\left(\right.\right.\right.$ lambdas. $\left.\left.{ }^{\wedge} 2-0.20692^{\wedge} 2\right)\right)+(100+2.657 \mathrm{e}-$

$5 * \mathrm{~F}) . /($ lambdas.^2-128.806)-0.015334*lambdas.^2);

nzI $=\operatorname{sqrt}\left(5.35583+4.629 \mathrm{e}-7 * \mathrm{~F}+\left(\left(0.100473+3.862 \mathrm{e}-8^{*} \mathrm{~F}\right) . /(\right.\right.$ lambdaI.^2-0.20692^2) $)+(100+2.657 \mathrm{e}-$

$5 * \mathrm{~F}) . /($ lambdaI.^2-128.806)-0.015334*lambdaI.^2);

deltak $=2 *$ pi $*($ nzp./lambdap-(nzs./lambdas+nzI./lambdaI)*cos(theta))-2*pi/32.93;\%condition of phase matching

efficiency $=(\operatorname{sinc}(\operatorname{deltak} * \mathrm{~L} / 2)) .^{\wedge} 2$;

figure(2); 
plot(lambdap,efficiency)

title('2 $\mathrm{cm}$ lithium niobate');

xlabel('pump wavelength(um)');

ylabel('quasi phase matching efficiency');

\section{Chapter III, 50/50 beam splitter}

clc;

clear all;

$\mathrm{i}=\operatorname{sqrt}(-1)$;

$\mathrm{n} 0=1.5$;\% beam spliter index

$\mathrm{n} 1=2.1+\mathrm{i} * 0.1 ; \%$ crack index

$\mathrm{n} 2=1.5 ; \%$ assume $\mathrm{n} 0=\mathrm{n} 2$, symmetry

$\mathrm{d}=0.5 \mathrm{e}-7: 0.001 \mathrm{e}-7: 3 \mathrm{e}-7$;

lambda $=0.8 \mathrm{e}-6$;

theta $0=\mathrm{pi} / 4$;

$\mathrm{k} 0=2 * \mathrm{pi}$ *n0/lambda;

$\mathrm{k} 1=2 * \mathrm{pi} * \mathrm{n} 1 /$ lambda;

$\mathrm{k} 2=2 * \mathrm{pi}$ *n2/lambda;

$\mathrm{kz} 0=\mathrm{k} 0 * \cos ($ theta 0$)$;

$\mathrm{kx} 0=\mathrm{k} 0 * \sin ($ theta 0$)$;

$\mathrm{kx} 1=\mathrm{kx} 0$;

$\mathrm{kz} 1=\operatorname{sqrt}\left(\mathrm{k} 0^{\wedge} 2 * \mathrm{n} 1^{\wedge} 2-\mathrm{kx} 1^{\wedge} 2\right) ;$

$\mathrm{kx} 2=\mathrm{kx} 0$;

$\mathrm{kz} 2=\operatorname{sqrt}\left(\mathrm{k} 0^{\wedge} 2^{*} \mathrm{n} 2^{\wedge} 2-\mathrm{kx} 2^{\wedge} 2\right) ;$

Einc=1;

Eref $=(1-\exp (-2 * \mathrm{i} * \mathrm{kz} 1 * \mathrm{~d})) * \operatorname{Einc} . /((\mathrm{kz} 1+\mathrm{kz} 0) /(\mathrm{kz} 1-\mathrm{kz} 0) * \exp (-2 * \mathrm{i} * \mathrm{kz} 1 * \mathrm{~d})-(\mathrm{kz} 1-\mathrm{kz} 0) /(\mathrm{kz} 1+\mathrm{kz} 0))$;

$\mathrm{Et} 1=$ Einc $* 4 * \mathrm{kz} 1 * \mathrm{kz} 0 . /\left((\mathrm{kz} 1+\mathrm{kz} 0)^{\wedge} 2 * \exp (-\mathrm{i} * \mathrm{kz} 1 * \mathrm{~d})-(\mathrm{kz} 1-\mathrm{kz} 0)^{\wedge} 2 * \exp (\mathrm{i} * \mathrm{kz} 1 * \mathrm{~d})\right)$;

$\mathrm{r}=($ Eref $) . /($ Einc $)$;

$\mathrm{R}=\operatorname{abs}(\mathrm{r}) .^{\wedge} 2$;

$\mathrm{t}=(\mathrm{Et} 1) \cdot /($ Einc) ;

$\mathrm{T}=\operatorname{abs}(\mathrm{t}) .^{\wedge} 2$;

\%subplot $(3,1,1)$; 


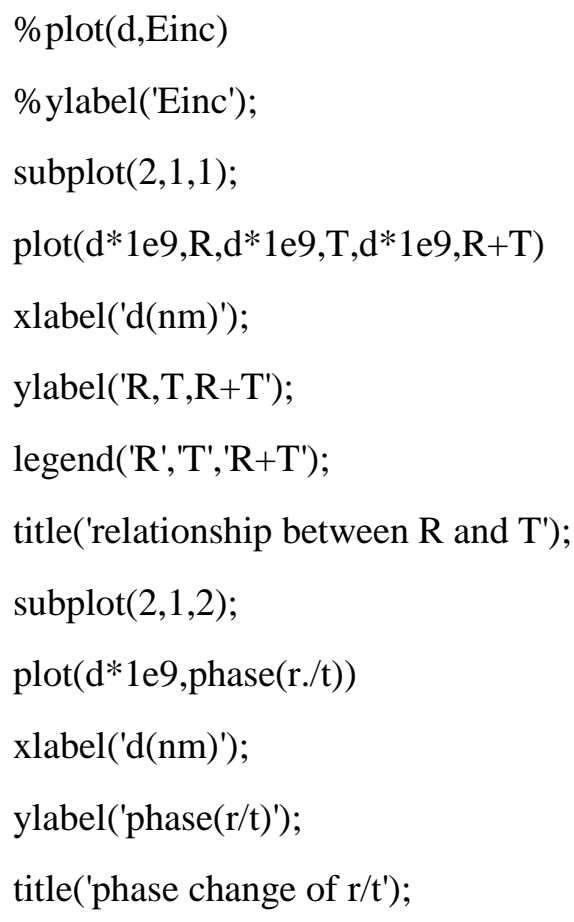

\section{(2) HOM visibility}

1. Visibility loss

clc; 
clear all;

close all;

$\mathrm{R}=0.5$;

$\mathrm{T}=0.4$;

deltat=-100:0.1:100;

deltaT=-100:5:100;

$\mathrm{N} 1=1600 *\left(\mathrm{~T}^{\wedge} 2+\mathrm{R}^{\wedge} 2\right)^{*}\left(1-\exp \left(-0.005 *\right.\right.$ deltat. $\left.\left.^{\wedge} 2\right)\right) ;$

$\mathrm{N} 2=1600 *\left(\mathrm{~T}^{\wedge} 2+\mathrm{R}^{\wedge} 2\right) *(1-\exp (-0.005 *$ deltaT.^2)$) ;$

$\mathrm{N} 3=1600 *\left(\mathrm{~T}^{\wedge} 2+\mathrm{R}^{\wedge} 2\right) *\left(1-0.9 * \exp \left(-0.005 *\right.\right.$ deltat. $\left.\left.^{\wedge} 2\right)\right)$;

$\mathrm{N} 4=1600 *\left(\mathrm{~T}^{\wedge} 2+\mathrm{R}^{\wedge} 2\right) *\left(1-0.75^{*} \exp \left(-0.005^{*}\right.\right.$ deltat. $\left.\left.{ }^{\wedge} 2\right)\right)$;

$\mathrm{N} 5=1600^{*}\left(\mathrm{~T}^{\wedge} 2+\mathrm{R}^{\wedge} 2\right)^{*}\left(1-0.6^{*} \exp \left(-0.005^{*}\right.\right.$ deltat. $\left.\left.^{\wedge} 2\right)\right)$;

figure(1);

plot(deltat,N1);

hold on;

scatter(deltaT,N2);

title('HOM dip simulation');

xlabel('BS position shift \mum');

ylabel('photon number of coincidence count');

legend('trend curve','coincidence distribution');

figure(2);

plot(deltat,N1);

hold on;

plot(deltat,N3);

hold on;

plot(deltat,N4);

hold on;

plot(deltat,N5);

title('Loss of Visibility');

xlabel('optical delay (fs)');

ylabel('photon number of coincidence count');

legend('T+R=1','T+R=0.9', 'T+R=0.75','T+R=0.6'); 


\section{Asymmetric wave dispersion}

clc;

clear all;

close all;

sigma $=25^{*} 10^{\wedge} 18$;

$\mathrm{W}=-100 * 10^{\wedge} 8: 0.01 * 10^{\wedge} 8: 100 * 10^{\wedge} 8 ;$

w2=-100:0.01:100;

$\mathrm{A} 1=1$;

$\mathrm{A} 2=0.6$;

$\% \mathrm{~A} 3=0.4$

$\mathrm{A} 4=0.1$;

fai $1=\exp (-\mathrm{w} . \wedge 2 / \operatorname{sigma}){ }^{*}\left(1-\mathrm{A} 1 * \sin \left(10^{\wedge}(-8)^{*} \mathrm{w}\right)\right)$;

$\operatorname{plot}(w, f a i 1)$;

hold on;

fai2=exp(-w.^2/sigma).*(1-A2* $\left.\sin \left(10^{\wedge}(-8)^{*} \mathrm{w}\right)\right)$;

$\operatorname{plot}(w, f a i 2)$;

\%hold on;

$\%$ fai3 $=\exp \left(-\mathrm{w} . \wedge 2 / \operatorname{sigma}^{*}{ }^{*}\left(1-\mathrm{A} 3{ }^{*} \sin \left(10^{\wedge}(-8)^{*} \mathrm{w}\right)\right)\right.$;

$\%$ plot(w,fai3);

hold on;

fai4=exp(-w.^2/sigma).*(1-A4* $\left.{ }^{*} \sin \left(10^{\wedge}(-8)^{*} \mathrm{w}\right)\right)$;

$\operatorname{plot}(w, f a i 4)$;

legend(' $\left.\mathrm{A}=1^{\prime},{ }^{\prime} \mathrm{A}=0.6^{\prime}, \mathrm{A}^{\prime} \mathrm{A}=0.1^{\prime}\right)$;

xlabel('osciliation frequency');

ylabel('Amplitude');

title(' Effect of sine modulation on a Gaussian wavepacket') 